\title{
Hybrid iterative scheme for a generalized equilibrium problems, variational inequality problems and fixed point problem of a finite family of $\kappa_{i}$-strictly pseudocontractive mappings
}

Atid Kangtunyakarn

Correspondence:

beawrock@hotmail.com

Department of Mathematics,

Faculty of Science, King Mongkut's

Institute of Technology

Ladkrabang, Bangkok 10520,

Thailand

\begin{abstract}
In this article, by using the S-mapping and hybrid method we prove a strong convergence theorem for finding a common element of the set of fixed point problems of a finite family of $\kappa_{i}$-strictly pseudocontractive mappings and the set of generalized equilibrium defined by Ceng et al., which is a solution of two sets of variational inequality problems. Moreover, by using our main result we have a strong convergence theorem for finding a common element of the set of fixed point problems of a finite family of $\kappa_{i}$-strictly pseudocontractive mappings and the set of solution of a finite family of generalized equilibrium defined by Ceng et al., which is a solution of a finite family of variational inequality problems.
\end{abstract}

Keywords: $k$-strict pseudo contraction mapping, $a$-inverse strongly monotone, generalized equilibrium problem, variational inequality, the S-mapping

\section{Introduction}

Let $H$ be a real Hilbert space and let $\mathrm{C}$ be a nonempty closed convex subset of $\mathrm{H}$. A mapping $T$ of $H$ into itself is called nonexpansive if $\|T x-T y\| \leq\|x-y\|$ for all $x, y \in H$. We denote by $F(T)$ the set of fixed points of $T$ (i.e., $F(T)=\{x \in H: T x=x\}$ ) Goebel and Kirk [1] showed that $F(T)$ is always closed convex, and also nonempty provided $T$ has a bounded trajectory.

Recall the mapping $T$ is said to be $\kappa$-strict pseudo-contration if there exist $\kappa \in[0,1)$ such that

$$
\|T x-T y\|^{2} \leq\|x-y\|^{2}+\kappa\|(I-T) x-(I-T) y\|^{2} \forall x, y \in D(T) .
$$

Note that the class of $\kappa$-strict pseudo-contractions strictly includes the class of nonexpansive mappings, that is $T$ is nonexpansive if and only if $\mathrm{T}$ is 0 -strict pseudo-contractive. If $\kappa=1, T$ is said to be pseudo-contraction mapping. $T$ is strong pseudo-contraction if there exists a positive constant $\lambda \in(0,1)$ such that $T+\lambda I$ is pseudo-contraction. In a real Hilbert space $H$ (1.1) is equivalent to

$$
\langle T x-T y, x-y\rangle \leq\|x-y\|^{2}-\frac{1-\kappa}{2}\|(I-T) x-(I-T) y\|^{2} \forall x, y \in D(T) .
$$

(c) 2012 Kangtunyakarn; licensee Springer. This is an Open Access article distributed under the terms of the Creative Commons Attribution License (http://creativecommons.org/licenses/by/2.0), which permits unrestricted use, distribution, and reproduction in any medium, provided the original work is properly cited. 
$T$ is pseudo-contraction if and only if

$$
\langle T x-T y, x-y\rangle \leq\|x-y\|^{2} \quad \forall x, y \in D(T) .
$$

$T$ is strong pseudo-contraction if there exists a positive constant $\lambda \in(0,1)$

$$
\langle T x-T y, x-y\rangle \leq(1-\lambda)\|x-y\|^{2} \quad \forall x, y \in D(T)
$$

The class of $\kappa$-strict pseudo-contractions falls into the one between classes of nonexpansive mappings and pseudo-contraction mappings and class of strong pseudo-contraction mappings is independent of the class of $\kappa$-strict pseudo-contraction.

A mapping $A$ of $C$ into $H$ is called inverse-strongly monotone, see [2] if there exists a positive real number $\alpha$ such that

$$
\langle x-y, A x-A y\rangle \geq \alpha\|A x-A y\|^{2}
$$

for all $x, y \in C$.

The equilibrium problem for $G$ is to determine its equilibrium points, i.e., the set

$$
E P(G)=\{x \in G: G(x, y) \geq 0, \quad \forall y \in C\} .
$$

Given a mapping $T: C \rightarrow H$, let $G(x, y)=\langle T x, y-x\rangle$ for all $x, y \in C$. Then, $z \in E P$ $(F)$ if and only if $\langle T z, y-z\rangle \geq 0$ for all $y \in C$, i.e., $z$ is a solution of the variational inequality. Let $A: C \rightarrow H$ be a nonlinear mapping. The variational inequality problem is to find a $u \in C$ such that

$$
\langle v-u, A u\rangle \geq 0
$$

for all $v \in C$. The set of solutions of the variational inequality is denoted by $\operatorname{VI}(C, A)$. In 2005, Combettes and Hirstoaga [3] introduced some iterative schemes of finding the best approximation to the initial data when $\mathrm{EP}(\mathrm{G})$ is nonempty and proved strong convergence theorem.

Also in [3] Combettes and Hiratoaga, following [4] define $S_{r}: H \rightarrow C$ by

$$
S_{r}(x)=\left\{z \in C: G(z, y)+\frac{1}{r}\langle y-z, z-x\rangle \geq 0 \forall y \in C\right\} .
$$

hey proved that under suitable hypotheses $G, S_{r}$ is single-valued and firmly nonexpansive with $F\left(S_{r}\right)=E P(G)$.

Numerous problems in physics, optimization, and economics reduce to find a element of $E P(G)$ (see, e.g., [5-16])

Let $C B(H)$ be the family of all nonempty closed bounded subsets of $H$ and $\mathcal{H}(.,$.$) be$ the Hausdorff metric on $\mathrm{CB}(\mathrm{H})$ defined as

$$
\mathcal{H}(U, V)=\max \left\{\sup _{u \in U} d(u, V), \sup _{v \in V} d(U, v)\right\}, \quad \forall U, V \in C B(H),
$$

where $d(u, V)=\inf _{v \in V} d(u, v), d(U, v)=\inf _{u \in U} d(u, v)$, and $d(u, v)=\|u-v\|$.

Let $C$ be a nonempty closed convex subset of $H$. Let $\phi: C \rightarrow \mathbb{R}$ be a real-valued function, $T: C \rightarrow C B(H)$ a multivalued mapping and $\Phi: H \times C \times C \rightarrow \mathbb{R}$ an equilibrium-like function, that is, $\Phi(w, u, v)+\Phi(w, v, u)=0$ for all $(w, u, v) \in H \times C \times C$ which satisfies the following conditions with respect to the multivalued map $T: C \rightarrow$ $C B(H)$. 
(H1) For each fixed $v \in C,(\omega, u) \mapsto \Phi(\omega, u, v)$ is an upper semicontinuous function from $H \times C$ to $\mathbb{R}$, that is, for $(\omega, u) \in H \times C$, whenever $\omega_{n} \rightarrow \omega$ and $u_{n} \rightarrow u$ as $n \rightarrow \infty$,

$$
\limsup _{n \rightarrow \infty} \Phi\left(\omega_{n}, u_{n}, v\right) \leq \Phi(\omega, u, v)
$$

(H2) For each fixed $(w, v) \in H \times C, u \mapsto \Phi(w, u, v)$ is a concave function;

(H3) For each fixed $(w, u) \in H \times C, v \mapsto \Phi(w, u, v)$ is a convex function.

In 2009, Ceng et al. [17] introduced the following generalized equilibrium problem (GEP) as follows:

$$
\text { (GEP) }\left\{\begin{array}{l}
\text { Find } u \in C \text { and } w \in T(u) \text { such that } \\
\Phi(w, u, v)+\varphi(v)-\varphi(u) \geq 0, \forall v \in C .
\end{array}\right.
$$

The set of such solutions $u \in C$ of $(G E P)$ is denote by $(G E P)_{s}(\Phi, \phi)$.

In the case of $\phi \equiv 0$ and $\Phi(w, u, v) \equiv G(u, v)$, then $(G E P)_{s}(\Phi, \phi)$ is denoted by $E P(G)$. By using Nadler's theorem they introduced the following algorithm:

Let $x_{1} \in C$ and $w_{1} \in T\left(x_{1}\right)$, there exists sequences $\left\{w_{n}\right\} \subseteq H$ and $\left\{x_{n}\right\},\left\{u_{n}\right\} \subseteq C$ such that

$$
\left\{\begin{array}{l}
w_{n} \in T\left(x_{n}\right),\left\|w_{n}-w_{n+1}\right\| \leq\left(1+\frac{1}{n}\right) \mathcal{H}\left(T\left(x_{n}\right), T\left(x_{n+1}\right)\right), \\
\Phi\left(w_{n}, u_{n}, v\right)+\varphi(v)-\varphi\left(u_{n}\right)+\frac{1}{r_{n}}\left\langle u_{n}-x_{n}, v-u_{n}\right\rangle \geq 0, \forall u \in C, \\
x_{n+1}=\alpha_{n} f\left(x_{n}\right)+\left(1-\alpha_{n}\right) S u_{n}, \quad n=1,2, \ldots .
\end{array}\right.
$$

They proved a strong convergence theorem of the sequence $\left\{x_{n}\right\}$ generated by (1.7) as follows:

Theorem 1.1. (See [17]) Let $C$ be a nonempty, bounded, closed, and convex subset of a real Hilbert space $H$ and let $\phi: C \rightarrow \mathbb{R}$ be a lower semicontinuous and convex functional. Let $T: C \rightarrow C B(H)$ be $\mathcal{H}$-Lipschitz continuous with constant $\mu, \Phi: H \times C \times C \rightarrow \mathbb{R}$ be an equilibrium-like function satisfying $(\mathrm{H1})-(\mathrm{H} 3)$ and $S$ be a nonexpansive mapping of $\mathrm{C}$ into itself such that $F(S) \cap(G E P)_{s}(\Phi, \varphi) \neq \emptyset$. Let $f$ be a contraction of $C$ into itself and let $\left\{x_{n}\right\}$, $\left\{w_{n}\right\}$, and $\left\{u_{n}\right\}$ be sequences generated by (1.7), where $\left\{\alpha_{n}\right\} \subseteq[0,1]$ and $\left\{r_{n}\right\} \subseteq(0, \infty)$ satisfy

$$
\begin{aligned}
& \lim _{n \rightarrow \infty} \alpha_{n}=0, \sum_{n=1}^{\infty} \alpha_{n}=\infty, \sum_{n=1}^{\infty}\left|\alpha_{n+1}-\alpha_{n}\right|<\infty, \\
& \liminf \operatorname{in}_{n \rightarrow \infty} r_{n}>0 \text { and } \sum_{n=1}^{\infty}\left|r_{n+1}-r_{n}\right|<\infty
\end{aligned}
$$

If there exists a constant $\lambda>0$ such that

$$
\Phi\left(w_{1}, T_{r_{1}}\left(x_{1}\right), T_{r_{2}}\left(x_{2}\right)\right)+\Phi\left(w_{2}, T_{r_{2}}\left(x_{2}\right), T_{r_{1}}\left(x_{1}\right)\right) \leq-\lambda\left\|T_{r_{1}}\left(x_{1}\right)-T_{r_{2}}\left(x_{2}\right)\right\|^{2}
$$

for all $\left(r_{1}, r_{2}\right) \in \Xi \times \Xi,\left(x_{1}, x_{2}\right) \in C \times C$ and $w_{i} \in T\left(x_{i}\right), i=1,2$, where $\Xi=\left\{r_{n}: n \geq 1\right\}$,

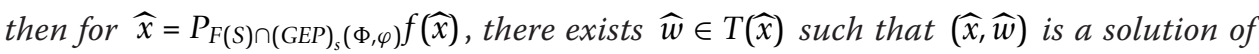
(GEP) and

$$
x_{n} \rightarrow \widehat{x}, w_{n} \rightarrow \widehat{w} \text { and } u_{n} \rightarrow \widehat{x} \text { as } n \rightarrow \infty .
$$

In 2011, Kangtunyakarn [18] proved the following theorem for strict pseudocontractive mapping in Hilbert space by using hybrid method as follows: 
Theorem 1.2. Let $C$ be a nonempty closed convex subset of a Hilbert space $H$. Let $F$ and $G$ be bifunctions from $C \times C$ into $\mathbb{R}$ satisfying $\left(A_{1}\right)-\left(A_{4}\right)$, respectively. Let $A: C \rightarrow$ $H$ be a $\alpha$-inverse strongly monotone mapping and let $B: C \rightarrow H$ be a $\beta$-inverse strongly monotone mapping. Let $T: C \rightarrow C$ be a $\kappa$-strict pseudo-contraction mapping with $\mathbb{F}=F(T) \cap E P(F, A) \cap E P(G, B) \neq \emptyset$. Let $\left\{x_{n}\right\}$ be a sequence generated by $x_{1} \in C=C_{1}$ and

$$
\left\{\begin{array}{l}
F\left(u_{n}, u\right)+\left(A x_{n}, u-u_{n}\right)+\frac{1}{r_{n}}\left\langle u-u_{n}, u_{n}-x_{n}\right\rangle \geq 0, \quad \forall u \in C, \\
G\left(v_{n}, v\right)+\left(B x_{n}, v-v_{n}\right)+\frac{1}{s_{n}}\left\langle v-v_{n}, v_{n}-x_{n}\right\rangle \geq 0, \quad \forall v \in C, \\
z_{n}=\delta_{n} u_{n}+\left(1-\delta_{n}\right) v_{n} \\
y_{n}=\alpha_{n} z_{n}+\left(1-\alpha_{n}\right) T z_{n} \\
C_{n+1}=\left\{z \in C_{n}:\left\|y_{n}-z\right\| \leq\left\|x_{n}-z\right\|\right\} \\
x_{n+1}=P_{C_{n+1}} x_{1}, \quad \forall n \geq 1
\end{array}\right.
$$

where $\left\{\alpha_{n}\right\}_{n=0}^{\infty}$ is sequence in $[0,1], r_{n} \in[a, b] \subset(0,2 \alpha)$ and $s_{n} \subset[c, d] \subset(0,2 \beta)$ satisfy the following condition:

$$
\begin{aligned}
& \text { (i) } \lim _{n \rightarrow \infty} \delta_{n}=\delta \in(0,1) \\
& \text { (ii) } 0 \leq \kappa \leq \alpha_{n}<1, \quad \forall n \geq 1
\end{aligned}
$$

Then $x_{n}$ converges strongly to $P_{\mathbb{F}} x_{1}$.

From motivation of (1.7) and (1.9), we define the following algorithm as follows:

Algorithm 1.3. Let $T_{i}, i=1,2, \ldots, N$, be $\kappa_{i}$-pseudo contraction mappings of $C$ into itself and $\kappa=\max \left\{\kappa_{i}: i=1,2, \ldots, N\right\}$ and let $S_{n}$ be the $S$-mappings generated by $T_{1}, T_{2}, \ldots, T_{N}$ and $\alpha_{1}^{(n)}, \alpha_{2}^{(n)}, \ldots, \alpha_{N}^{(n)}$ where $\alpha_{j}^{(n)}=\left(\alpha_{1}^{n, j}, \alpha_{2}^{n, j}, \alpha_{3}^{n, j}\right) \in I \times I \times I, I=[0,1], \alpha_{1}^{n, j}+\alpha_{2}^{n, j}+\alpha_{3}^{n, j}=1$ and $\kappa<a \leq \alpha_{1}^{n, j}, \alpha_{3}^{n, j} \leq b<1$ for all $j=1,2, \ldots, N-1, \kappa<c \leq \alpha_{1}^{n, N} \leq 1, \kappa \leq \alpha_{3}^{n, N} \leq d<1, \kappa \leq \alpha_{2}^{n, j} \leq e<1$ for all $j=1,2, \ldots, N$. Let $x_{1} \in C=C_{1}$ and $w_{1}^{1} \in T\left(x_{1}\right), w_{1}^{2} \in D\left(x_{1}\right)$, there exists sequence $\left\{w_{n}^{1}\right\},\left\{w_{n}^{2}\right\} \in H$ and $\left\{x_{n}\right\},\left\{u_{n}\right\},\left\{v_{n}\right\} \subseteq C$ such that

$$
\left\{\begin{array}{l}
w_{n}^{1} \in T\left(x_{n}\right), \quad\left\|w_{n}^{1}-w_{n+1}^{1}\right\| \leq\left(1+\frac{1}{n}\right) \mathcal{H}\left(T\left(x_{n}\right), T\left(x_{n+1}\right)\right), \\
w_{n}^{2} \in D\left(x_{n}\right),\left\|w_{n}^{2}-w_{n+1}^{2}\right\| \leq\left(1+\frac{1}{n}\right) \mathcal{H}\left(D\left(x_{n}\right), D\left(x_{n+1}\right)\right), \\
\Phi_{1}\left(w_{n}^{1}, u_{n}, u\right)+\varphi_{1}(u)-\varphi_{1}\left(u_{n}\right)+\frac{1}{r_{n}}\left\langle u_{n}-x_{n}, u-u_{n}\right\rangle \geq 0, \quad \forall u \in C \\
\Phi_{2}\left(w_{n}^{2}, v_{n}, v\right)+\varphi_{2}(v)-\varphi_{2}\left(v_{n}\right)+\frac{1}{s_{n}}\left\langle v_{n}-x_{n}, v-v_{n}\right\rangle \geq 0, \quad \forall v \in C \\
z_{n}=\delta_{n} P_{C}(I-\lambda A) u_{n}+\left(1-\delta_{n}\right) P_{C}(I-\eta B) v_{n}, \\
y_{n}=\alpha_{n} z_{n}+\left(1-\alpha_{n}\right) S_{n} z_{n} \\
C_{n+1}=\left\{z \in C_{n}:\left\|y_{n}-z\right\| \leq\left\|x_{n}-z\right\|\right\} \\
x_{n+1}=P_{C_{n+1}} x_{1}, \quad \forall n \geq 1 .
\end{array}\right.
$$

where $D, T: C \rightarrow C B(H)$ are $\mathcal{H}$-Lipschitz continuous with constant $\mu_{1}, \mu_{2}$, respectively, $\Phi_{1}, \Phi_{2}: H \times C \times C \rightarrow \mathbb{R}$ are equilibrium-like functions satisfying (H1)-(H3), $A$ : 
$C \rightarrow H$ is a $\alpha$-inverse strongly monotone mapping and $B: C \rightarrow H$ is a $\beta$-inverse strongly monotone mapping.

In this article, we prove under some control conditions on $\left\{\delta_{n}\right\},\left\{\alpha_{n}\right\},\left\{s_{n}\right\}$, and $\left\{r_{n}\right\}$ that the sequence $\left\{x_{n}\right\}$ generated by (1.7) converges strongly to $P_{\mathbb{E}} x_{1}$ where $\mathbb{F}=\cap_{i=1}^{N} F\left(T_{i}\right) \cap(G E P)_{s}\left(\Phi_{1}, \varphi_{1}\right) \cap(G E P)_{s}\left(\Phi_{2}, \varphi_{2}\right) \cap F\left(G_{1}\right) \cap F\left(G_{2}\right), G_{1}, G_{2}: C \rightarrow C$ are defined by $G_{1}(x)=P_{C}(x-\lambda A x), G_{2}(x)=P_{C}(x-\eta B x), \forall x \in C$ and $P_{\mathbb{F}} x_{1}$ is solution of the following system of variational inequality:

$$
\left\{\begin{array}{l}
\left\langle A x^{*}, x-x^{*}\right\rangle \geq 0 \\
\left\langle B x^{*}, x-x^{*}\right\rangle \geq 0
\end{array}\right.
$$

\section{Preliminaries}

In this section, we need the following lemmas and definition to prove our main result.

Let $C$ be a nonempty closed convex subset of $H$. Then for any $x \in H$, there exists a unique nearest point in $C$, denoted by $P_{C} x$, such that

$$
\left\|x-P_{C} x\right\| \leq\|x-y\|, \text { for all } y \in C .
$$

The following lemma is a property of $P_{C}$.

Lemma 2.1. (See [19].) Given $x \in H$ and $y \in C$. Then $P_{C} x=y$ if and only if there holds the inequality

$$
\langle x-y, y-z\rangle \geq 0 \quad \forall z \in C
$$

Lemma 2.2. (See [20]) Let $C$ be a closed convex subset of a strictly convex Banach space E. Let $\left\{T_{n}: n \in \mathbb{N}\right\}$ be a sequence of nonexpansive mappings on C. Suppose $\cap_{n=1}^{\infty} F\left(T_{n}\right)$ is nonempty. Let $\left\{\lambda_{n}\right\}$ be a sequence of positive numbers with $\sum_{n=1}^{\infty} \lambda_{n}=1$.

Then a mapping $S$ on $C$ defined by

$$
S(x)=\sum_{n=1}^{\infty} \lambda_{n} T_{n} x
$$

for $x \in C$ is well defined, nonexpansive and $F(S)=\cap_{n=1}^{\infty} F\left(T_{n}\right)$ hold.

The following lemma is well known.

Lemma 2.3. Let $H$ be Hilbert space, $C$ be a nonempty closed convex subset of H. Let $T: C \rightarrow C$ be a $\kappa$-strictly pseudo-contractive, then the fixed point set $F(T)$ of $T$ is closed and convex so that the projection $P_{F(T)}$ is well defined.

In 2009, Kangtunyakarn and Suantai [21] introduced the $S$-mapping generated by a finite family of $\kappa$-strictly pseudo contractive mappings and real numbers as follows: 
Definition 2.1. Let $C$ be a nonempty convex subset of real Hilbert space. Let $\left\{T_{i}\right\}_{i=1}^{N}$ be a finite family of $\kappa_{i}$-strict pseudo-contractions of $C$ into itself. For each $j=1,2, \ldots, N$, let $\alpha_{j}=\left(a_{1}^{j}, \alpha_{2}^{j}, \alpha_{3}^{j}\right) \in I \times I \times I$ where $I \in[0,1]$ and $\alpha_{1}^{j}+\alpha_{2}^{j}+\alpha_{3}^{j}=1$. We define the mapping $S: C \rightarrow C$ as follows:

$$
\begin{aligned}
U_{0} & =I \\
U_{1} & =\alpha_{1}^{1} T_{1} U_{0}+\alpha_{2}^{1} U_{0}+\alpha_{3}^{1} I \\
U_{2} & =\alpha_{1}^{2} T_{2} U_{1}+\alpha_{2}^{2} U_{1}+\alpha_{3}^{2} I \\
U_{3} & =\alpha_{1}^{3} T_{3} U_{2}+\alpha_{2}^{3} U_{2}+\alpha_{3}^{3} I \\
& \cdot \\
\cdot & \\
\cdot & \\
U_{N-1} & =\alpha_{1}^{N-1} T_{N-1} U_{N-2}+\alpha_{2}^{N-1} U_{N-2}+\alpha_{3}^{N-1} I \\
S & =U_{N}=\alpha_{1}^{N} T_{N} U_{N-1}+\alpha_{2}^{N} U_{N-1}+\alpha_{3}^{N} I .
\end{aligned}
$$

This mapping is called $S$-mapping generated by $T_{1}, \ldots, T_{N}$ and $\alpha_{1}, \alpha_{2}, \ldots, \alpha_{N}$.

Lemma 2.4. (See [21]) Let $C$ be a nonempty closed convex subset of real Hilbert space. Let $\left\{T_{i}\right\}_{i=1}^{N}$ be a finite family of $\kappa$-strict pseudo contraction mapping of $C$ into $C$ with $\cap_{i=1}^{N} F\left(T_{i}\right) \neq \emptyset$ and $\kappa=\max \left\{\kappa_{i}: i=1,2, \ldots, N\right\}$ and let $\alpha_{j}=\left(a_{1}^{j}, \alpha_{2}^{j}, \alpha_{3}^{j}\right) \in I \times I \times I$, $j=1,2,3, \ldots, N$, where $I=[0,1], \alpha_{1}^{j}+\alpha_{2}^{j}+\alpha_{3}^{j}=1, \alpha_{1}^{j}, \alpha_{3}^{j} \in(\kappa, 1)$ for all $j=1,2, \ldots, N-1$ and $\alpha_{1}^{N} \in(\kappa, 1], \alpha_{3}^{N} \in[\kappa, 1) \alpha_{2}^{j} \in[\kappa, 1)$ for all $j=1,2, \ldots, N$. Let $S$ be the mapping generated by $T_{1}, \ldots . ., T_{N}$ and $\alpha_{1}, \alpha_{2}, \ldots, \alpha_{N}$. Then $F(S)=\cap_{i=1}^{N} F\left(T_{i}\right)$ and $S$ is a nonexpansive mapping.

Lemma 2.5. (See [22]) Let $C$ be a nonempty closed convex subset of a real Hilbert space $H$ and $S: C \rightarrow C$ be a self-mapping of $C$. If $S$ is a $\kappa$-strict pseudo-contraction mapping, then $S$ satisfies the Lipschitz condition

$$
\|S x-S y\| \leq \frac{1+\kappa}{1-\kappa}\|x-y\|, \quad \forall x, \quad y \in C .
$$

We prove the following lemma by using the concept of the $S$-mapping as follows:

Lemma 2.6. Let $C$ be a nonempty closed convex subset of a real Hilbert space H. Let $T_{i}, i=1,2, \ldots, N$ be $\kappa_{i}$ strictly pseudo-contraction mappings of $C$ into itself and $\kappa=\max$ $\left\{\kappa_{i}: i=1,2, \ldots, N\right\}$ and let $\alpha_{j}^{(n)}=\left(\alpha_{1}^{n, j}, \alpha_{2}^{n, j}, \alpha_{3}^{n, j}\right), \alpha_{j}=\left(\alpha_{1}^{j}, \alpha_{2}^{j}, \alpha_{3}^{j}\right) \in I \times I \times I$, where $I=[0,1], \alpha_{1}^{n, j}+\alpha_{2}^{n, j}+\alpha_{3}^{n, j}=1$ and $\alpha_{1}^{j}+\alpha_{2}^{j}+\alpha_{3}^{j}=1$ such that $\alpha_{i}^{n, j} \rightarrow \alpha_{i}^{j} \in[0,1]$ as $n$ $\rightarrow \infty$ for $i=1,3$ and $j=1,2,3, \ldots, N$. For every $n \in \mathbb{N}$, let $S$ and $S_{n}$ be the S-mapping generated by $T_{1}, T_{2}, \ldots, T_{N}$ and $\alpha_{1}, \alpha_{2}, \ldots, \alpha_{N}$ and $T_{1}, T_{2}, \ldots, T_{N}$ and $\alpha_{1}^{(n)}, \alpha_{2}^{(n)}, \ldots, \alpha_{N}^{(n)}$, respectively. Then $\lim _{n \rightarrow \infty}\left\|S_{n} x_{n}-S x_{n}\right\|=0$ for every bounded sequence $\left\{x_{n}\right\}$ in $C$.

Proof. Let $\left\{x_{n}\right\}$ be bounded sequence in $C, U_{k}$ and $U_{n, k}$ be generated by $T_{1}, T_{2}, \ldots, T_{N}$ and $\alpha_{1}, \alpha_{2}, \ldots, \alpha_{N}$ and $T_{1}, T_{2}, \ldots, T_{N}$ and $\alpha_{1}^{(n)}, \alpha_{2}^{(n)}, \ldots, \alpha_{N}^{(n)}$, respectively. For each $n \in \mathbb{N}$, we have 


$$
\begin{aligned}
\left\|U_{n, 1} x_{n}-U_{1} x_{n}\right\| & =\left\|\alpha_{1}^{n, 1} T_{1} x_{n}+\left(1-\alpha_{1}^{n, 1}\right) x_{n}-\alpha_{1}^{1} T_{1} x_{n}-\left(1-\alpha_{1}^{1}\right) x_{n}\right\| \\
& =\left\|\alpha_{1}^{n, 1} T_{1} x_{n}-\alpha_{1}^{n, 1} x_{n}-\alpha_{1}^{1} T_{1} x_{n}+\alpha_{1}^{1} x_{n}\right\| \\
& =\left\|\left(\alpha_{1}^{n, 1}-\alpha_{1}^{1}\right) T_{1} x_{n}-\left(\alpha_{1}^{n, 1}-\alpha_{1}^{1}\right) x_{n}\right\| \\
& =\left|a_{1}^{n, 1}-\alpha_{1}^{1}\right|\left\|T_{1} x_{n}-x_{n}\right\|
\end{aligned}
$$

and for $k \in\{2,3, \ldots, N\}$, by using Lemma 2.5 , we obtain

$$
\begin{aligned}
& \left\|U_{n, k} x_{n}-U_{k} x_{n}\right\|=\| \alpha_{1}^{n, k} T_{k} U_{n, k-1} x_{n}+\alpha_{2}^{n, k} U_{n, k-1} x_{n}+\alpha_{3}^{n, k} x_{n} \\
& -\alpha_{1}^{k} T_{k} U_{k-1} x_{n}-\alpha_{2}^{k} U_{k-1} x_{n}-\alpha_{3}^{k} x_{n} \| \\
& =\| \alpha_{1}^{n, k} T_{k} U_{n, k-1} x_{n}+\alpha_{3}^{n, k} x_{n}-\alpha_{1}^{k} T_{k} U_{k-1} x_{n}-\alpha_{3}^{k} x_{n} \\
& +\alpha_{2}^{n, k} U_{n, k-1} x_{n}-\alpha_{2}^{k} U_{k-1} x_{n} \| \\
& =\| \alpha_{1}^{n, k} T_{k} U_{n, k-1} x_{n}-\alpha_{1}^{n, k} T_{k} U_{k-1} x_{n}+\alpha_{1}^{n, k} T_{k} U_{k-1} x_{n} \\
& -\alpha_{1}^{k} T_{k} U_{k-1} x_{n}+\left(\alpha_{3}^{n, k}-\alpha_{3}^{k}\right) x_{n}+\alpha_{2}^{n, k} U_{n, k-1} x_{n}-\alpha_{2}^{k} U_{k-1} x_{n} \| \\
& =\| \alpha_{1}^{n, k}\left(T_{k} U_{n, k-1} x_{n}-T_{k} U_{k-1} x_{n}\right)+\left(\alpha_{1}^{n, k}-\alpha_{1}^{k}\right) T_{k} U_{k-1} x_{n} \\
& +\left(\alpha_{3}^{n, k}-\alpha_{3}^{k}\right) x_{n}+\alpha_{2}^{n, k} U_{n, k-1} x_{n}-\alpha_{2}^{k} U_{k-1} x_{n} \| \\
& =\| \alpha_{1}^{n, k}\left(T_{k} U_{n, k-1} x_{n}-T_{k} U_{k-1} x_{n}\right)+\left(\alpha_{1}^{n, k}-\alpha_{1}^{k}\right) T_{k} U_{k-1} x_{n} \\
& +\left(\alpha_{3}^{n, k}-\alpha_{3}^{k}\right) x_{n}+\alpha_{2}^{n, k} U_{n, k-1} x_{n}-\alpha_{2}^{n, k} U_{k-1} x_{n} \\
& +\alpha_{2}^{n, k} U_{k-1} x_{n}-\alpha_{2}^{k} U_{k-1} x_{n} \| \\
& =\| \alpha_{1}^{n, k}\left(T_{k} U_{n, k-1} x_{n}-T_{k} U_{k-1} x_{n}\right)+\left(\alpha_{1}^{n, k}-\alpha_{1}^{k}\right) T_{k} U_{k-1} x_{n} \\
& +\left(\alpha_{3}^{n, k}-\alpha_{3}^{k}\right) x_{n}+\alpha_{2}^{n, k}\left(U_{n, k-1} x_{n}-U_{k-1} x_{n}\right) \\
& +\left(\alpha_{2}^{n, k}-\alpha_{2}^{k}\right) U_{k-1} x_{n} \| \\
& \leq \alpha_{1}^{n, k}\left\|T_{k} U_{n, k-1} x_{n}-T_{k} U_{k-1} x_{n}\right\|+\left|\alpha_{1}^{n, k}-\alpha_{1}^{k}\right|\left\|T_{k} U_{k-1} x_{n}\right\| \\
& +\left|\alpha_{3}^{n, k}-\alpha_{3}^{k}\right|\left\|x_{n}\right\|+\alpha_{2}^{n, k}\left\|U_{n, k-1} x_{n}-U_{k-1} x_{n}\right\| \\
& +\left|\alpha_{2}^{n, k}-\alpha_{2}^{k}\right|\left\|U_{k-1} x_{n}\right\| \\
& =\alpha_{1}^{n, k}\left\|T_{k} U_{n, k-1} x_{n}-T_{k} U_{k-1} x_{n}\right\|+\left|\alpha_{1}^{n, k}-\alpha_{1}^{k}\right|\left\|T_{k} U_{k-1} x_{n}\right\| \\
& +\alpha_{2}^{n, k}\left\|U_{n, k-1} x_{n}-U_{k-1} x_{n}\right\|+\mid 1-\alpha_{1}^{n, k}-\alpha_{3}^{n, k}-1 \\
& +\alpha_{1}^{k}+\alpha_{3}^{k}\left|\left\|U_{k-1} x_{n}\right\|+\right| \alpha_{3}^{n, k}-\alpha_{3}^{k} \mid\left\|x_{n}\right\| \\
& \leq \alpha_{1}^{n, k} \frac{1+\kappa}{1-\kappa}\left\|U_{n, k-1} x_{n}-U_{k-1} x_{n}\right\|+\mid \alpha_{1}^{n, k} \\
& -\alpha_{1}^{k} \mid\left\|T_{k} U_{k-1} x_{n}\right\|+\alpha_{2}^{n, k}\left\|U_{n, k-1} x_{n}-U_{k-1} x_{n}\right\|+\left(\left|\alpha_{1}^{k}-\alpha_{1}^{n, k}\right|\right. \\
& \left.+\left|\alpha_{3}^{n, k}-\alpha_{3}^{k}\right|\right)\left\|U_{k-1} x_{n}\right\|+\left|\alpha_{3}^{n, k}-\alpha_{3}^{k}\right|\left\|x_{n}\right\| \\
& \leq \frac{1+\kappa}{1-\kappa}\left\|U_{n, k-1} x_{n}-U_{k-1} x_{n}\right\|+\left|\alpha_{1}^{n, k}-\alpha_{1}^{k}\right|\left\|T_{k} U_{k-1} x_{n}\right\| \\
& +\frac{1-\kappa}{1-\kappa}\left\|U_{n, k-1} x_{n}-U_{k-1} x_{n}\right\|+\left(\left|\alpha_{1}^{k}-\alpha_{1}^{n, k}\right|\right. \\
& \left.+\left|\alpha_{3}^{n, k}-\alpha_{3}^{k}\right|\right)\left\|U_{k-1} x_{n}\right\|+\left|\alpha_{3}^{n, k}-\alpha_{3}^{k}\right|\left\|x_{n}\right\| \\
& \leq \frac{2}{1-\kappa}\left\|U_{n, k-1} x_{n}-U_{k-1} x_{n}\right\|+\left|\alpha_{1}^{n, k}-\alpha_{1}^{k}\right|\left(\left\|T_{k} U_{k-1} x_{n}\right\|+\left\|U_{k-1} x_{n}\right\|\right) \\
& +\left|\alpha_{3}^{n, k}-\alpha_{3}^{k}\right|\left(\left\|U_{k-1} x_{n}\right\|+\left\|x_{n}\right\|\right) \text {. }
\end{aligned}
$$


By (2.2) and (2.3), we have

$$
\begin{aligned}
& \left\|S_{n} x_{n}-S x_{n}\right\|=\left\|U_{n, N} x_{n}-U_{N} x_{n}\right\| \\
& \leq \frac{2}{1-\kappa}\left\|U_{n, N-1} x_{n}-U_{N-1} x_{n}\right\|+\left|\alpha_{1}^{n, N}-\alpha_{1}^{N}\right|\left(\left\|T_{N} U_{N-1} x_{n}\right\|\right. \\
& \left.+\left\|U_{N-1} x_{n}\right\|\right)+\left|\alpha_{3}^{n, N}-\alpha_{3}^{N}\right|\left(\left\|U_{N-1} x_{n}\right\|+\left\|x_{n}\right\|\right) \\
& \leq \frac{2}{1-\kappa}\left(\frac{2}{1-\kappa}\left\|U_{n, N-2} x_{n}-U_{N-2} x_{n}\right\|+\mid \alpha_{1}^{n, N-1}\right. \\
& -\alpha_{1}^{N-1} \mid\left(\left\|T_{N-1} U_{N-2} x_{n}\right\|\right. \\
& \left.\left.+\left\|U_{N-2} x_{n}\right\|\right)+\left|\alpha_{3}^{n, N-1}-\alpha_{3}^{N-1}\right|\left(\left\|U_{N-2} x_{n}\right\|+\left\|x_{n}\right\|\right)\right) \\
& +\left|\alpha_{1}^{n, N}-\alpha_{1}^{N}\right|\left(\left\|T_{N} U_{N-1} x_{n}\right\|+\left\|U_{N-1} x_{n}\right\|\right) \\
& +\left|\alpha_{3}^{n, N}-\alpha_{3}^{N}\right|\left(\left\|U_{N-1} x_{n}\right\|+\left\|x_{n}\right\|\right) \\
& =\left(\frac{2}{1-\kappa}\right)^{2}\left\|U_{n, N-2} x_{n}-U_{N-2} x_{n}\right\|+\sum_{j=N-1}^{N}\left(\frac{2}{1-\kappa}\right)^{N-j} \mid \alpha_{1}^{n, j} \\
& -\alpha_{1}^{j} \mid\left(\left\|T_{j} U_{j-1} x_{n}\right\|+\left\|U_{j-1} x_{n}\right\|\right) \\
& +\sum_{j=N-1}^{N}\left(\frac{2}{1-\kappa}\right)^{N-j}\left|\alpha_{3}^{n, j}-\alpha_{3}^{j}\right|\left(\left\|U_{j-1} x_{n}\right\|+\left\|x_{n}\right\|\right) \\
& \leq\left(\frac{2}{1-\kappa}\right)^{N-1}\left\|U_{n, 1} x_{n}-U_{1} x_{n}\right\|+\sum_{j=2}^{N}\left(\frac{2}{1-\kappa}\right)^{N-j} \mid \alpha_{1}^{n, j} \\
& -\alpha_{1}^{j}\left|\left(\left\|T_{j} U_{j-1} x_{n}\right\|+\left\|U_{j-1} x_{n}\right\|\right)+\sum_{j=2}^{N}\left(\frac{2}{1-\kappa}\right)^{N-j}\right| \alpha_{3}^{n, j}-\alpha_{3}^{j} \mid\left(\left\|U_{j-1} x_{n}\right\|+\left\|x_{n}\right\|\right) \\
& =\left(\frac{2}{1-\kappa}\right)^{N-1}\left|\alpha_{1}^{n, 1}-\alpha_{1}^{1}\right|\left\|T_{1} x_{n}-x_{n}\right\|+\sum_{j=2}^{N}\left(\frac{2}{1-\kappa}\right)^{N-j} \mid \alpha_{1}^{n, j} \\
& -\alpha_{1}^{j} \mid\left(\left\|T_{j} U_{j-1} x_{n}\right\|+\left\|U_{j-1} x_{n}\right\|\right) \\
& +\sum_{j=2}^{N}\left(\frac{2}{1-\kappa}\right)^{N-j}\left|\alpha_{3}^{n, j}-\alpha_{3}^{j}\right|\left(\left\|U_{j-1} x_{n}\right\|+\left\|x_{n}\right\|\right) \text {. }
\end{aligned}
$$

This together with the assumption $\alpha_{i}^{n, j} \rightarrow \alpha_{i}^{j}$ as $n \rightarrow \infty(i=1,3, j=1,2, \ldots, N)$, we can conclude that

$$
\lim _{n \rightarrow \infty}\left\|S_{n} x_{n}-S x_{n}\right\|=0
$$

Lemma 2.7. (See [23]) Let E be a uniformly convex Banach space, $C$ be a nonempty closed convex subset of $E$ and $S: C \rightarrow C$ be a nonexpansive mapping. Then $I-S$ is demi-closed at zero.

Lemma 2.8. (See [24]) Let $C$ be a closed convex subset of $H$. Let $\left\{x_{n}\right\}$ be a sequence in $H$ and $u \in H$. Let $q=P_{C} u$, if $\left\{x_{n}\right\}$ is such the $\omega\left(x_{n}\right) \subset C$ and satisfy the condition

$$
\left\|x_{n}-u\right\| \leq\|u-q\|, \quad \forall n \in \mathbb{N} .
$$

Then $x_{n} \rightarrow q$, as $n \rightarrow \infty$.

Definition 2.2. A multivalued map $T: C \rightarrow C B(H)$ is say to be $\mathcal{H}$-Lipschitz continuous if there exists a constant $\mu>0$ such that 


$$
\mathcal{H}(T(u)-T(v)) \leq \mu\|u-v\|, \quad \forall u, v \in C,
$$

where $\mathcal{H}(.,$.$) is the Hausdorff metric on C B(H)$.

Lemma 2.9. (Nadler's theorem, see [25]) Let $(X,\|\cdot\|)$ be a normed vector space and $\mathcal{H}(.,$.$) is the Hausdorff metric on C B(H)$. If $U, V \in C B(X)$, then for any given $\epsilon>0$ and $u \in U$, there exists $v \in V$ such that

$$
\|u-v\| \leq(1+\varepsilon) \mathcal{H}(U, V) .
$$

Let $C$ be a nonempty closed convex subset of a real Hilbert space $H$. Let $\phi: C \rightarrow H$ be a real-valued function, $T: C \rightarrow C B(H)$ be a multivalued map and $\Phi: H \times C \times C \rightarrow$ $\mathbb{R}$ be an equilibrium-like function.

To solve the GEP, let us assume that the equilibrium-like function $\Phi: H \times C \times C \rightarrow$ $\mathbb{R}$ satisfies the following conditions with respect to the multivalued map $T: C \rightarrow C B$ $(H)$.

(H1) For each fixed $v \in C,(\omega, u) \mapsto \Phi(\omega, u, v)$ is an upper semicontinuous function from $H \times C$ to $\mathbb{R}$, that is, for $(\omega, u) \in H \times C$, whenever $\omega_{n} \rightarrow \omega$ and $u_{n} \rightarrow u$ as $n \rightarrow$ $\infty$,

$$
\limsup _{n \rightarrow \infty} \Phi\left(\omega_{n}, u_{n}, v\right) \leq \Phi(\omega, u, v)
$$

(H2) For each fixed $(w, v) \in H \times C, u \mapsto \Phi(w, u, v)$ is a concave function;

(H3) For each fixed $(w, u) \in H \times C, v \mapsto \Phi(w, u, v)$ is a convex function.

Theorem 2.10. (See [17]) Let $C$ be a nonempty, bounded, closed, and convex subset of a real Hilbert space $H$, and let $\phi: C \rightarrow \mathbb{R}$ be a lower semicontinuous and convex functional. Let $T: C \rightarrow C B(H)$ be $\mathcal{H}$-Lipschitz continuous with constant $\mu$, and $\Phi: H \times C$ $\times C \rightarrow \mathbb{R}$ be an equilibrium-like function satisfying (H1)-(H3). Let $r>0$ be a constant. For each $x \in C$, take $w_{x} \in T(x)$ arbitrarily and define a mapping $T_{r}: C \rightarrow C$ as follows:

$$
T_{r}(x)=\left\{u \in C: \Phi\left(w_{x}, u, v\right)+\varphi(v)-\varphi(u)+\frac{1}{r}\langle u-x, v-u\rangle \geq 0, \quad \forall v \in C\right\} .
$$

Then, there hold the following:

(a) $T_{r}$ is single-valued;

(b) $T_{r}$ is firmly nonexpansive (that is, for any $u, v \in C,\left\|T_{r} u-T_{r} v\right\|^{2} \leq\left\langle T_{r} u-T_{r} v, u-v\right\rangle$ ) if

$$
\Phi\left(w_{1}, T_{r}\left(x_{1}\right), T_{r}\left(x_{2}\right)\right)+\Phi\left(w_{2}, T_{r}\left(x_{2}\right), T_{r}\left(x_{1}\right)\right) \leq 0,
$$

for all $\left(x_{1}, x_{2}\right) \in C \times C$ and all $w_{i} \in T\left(x_{\mathrm{i}}\right), i=1,2$;

(c) $F\left(T_{r}\right)=(G E P)_{s}(\Phi, \phi)$

(d) $(G E P)_{s}(\Phi, \phi)$ is closed and convex.

Lemma 2.11. (See [26]) Let $C$ be a nonempty closed convex subset of a Hilbert space $H$ and let $G: C \rightarrow C$ be defined by

$$
G(x)=P_{C}(x-\lambda A x), \quad \forall x \in C,
$$

with $\forall \lambda>0$. Then $x^{*} \in V I(C, A)$ if and only if $x^{*} \in F(G)$. 


\section{Main results}

In this section, we prove a strong convergence theorem of the sequence $\left\{x_{n}\right\}$ generated by $(1.10)$ to $P_{\mathbb{E}} x_{1}$.

Theorem 3.1. Let $C$ be a nonempty bounded, closed, and convex subset of Hilbert space $H$ and let $\phi_{1}, \phi_{2}$ : be a lower semicontinuous and convex function. Let $D, T: C \rightarrow C B(H)$ be $\mathcal{H}$-Lipschitz continuous with constant $\mu_{1}, \mu_{2}$, respectively, $\Phi_{1}, \Phi_{2}: H \times C \times C \rightarrow \mathbb{R}$ be equilibrium-like functions satisfying (H1) - (H3). Let $A: C \rightarrow H$ be a $\alpha$-inverse strongly monotone mapping and $B: C \rightarrow H$ be a $\beta$-inverse strongly monotone mapping, let $T_{i}, i=$ $1,2, \ldots, N$, be $\kappa_{i}$-pseudo contraction mappings of $C$ into itself and $\kappa=\max \left\{\kappa_{i}: i=1,2, \ldots, N\right\}$ with $\mathbb{F}=\cap_{i=1}^{N} F\left(T_{i}\right) \cap(G E P)_{s}\left(\Phi_{1}, \varphi_{1}\right) \cap(G E P)_{s}\left(\Phi_{2}, \varphi_{2}\right) \cap F\left(G_{1}\right) \cap F\left(G_{2}\right)$, where $G_{1}, G_{2}$ : $C \rightarrow C$ are defined by $G_{1}(x) P_{C}(x-\lambda A x), G_{2}(x)=P_{C}(x-\eta B x), \forall x \in C$. Let $S_{n}$ be the S-mappings generated by $T_{1}, T_{2}, \ldots, T_{N}$ and $\alpha_{1}^{(n)}, \alpha_{2}^{(n)}, \ldots, \alpha_{N}^{(n)} \quad$ where $\alpha_{j}^{(n)}=\left(\alpha_{1}^{n, j}, \alpha_{2}^{n, j}, \alpha_{3}^{n, j}\right) \in I \times I \times I, I=[0,1], \alpha_{1}^{n, j}+\alpha_{2}^{n, j}+\alpha_{3}^{n, j}=1$ and $\kappa<a \leq \alpha_{1}^{n, j}, \alpha_{3}^{n, j} \leq b<1$ for all $j=1,2, \ldots, N-1, \kappa<c \leq \alpha_{1}^{n, N} \leq 1, \kappa \leq \alpha_{3}^{n, N} \leq d<1, \kappa \leq \alpha_{2}^{n, j} \leq e<1$ for all $j$ $=1,2, \ldots, N$ and let $\left\{x_{n}\right\},\left\{u_{n}\right\},\left\{v_{n}\right\},\left\{w_{n}^{1}\right\}$, and $\left\{w_{n}^{2}\right\}$ be sequences generated by (1.10), where $\left\{\alpha_{n}\right\}$ is a sequence in $[0,1], r_{n}, \lambda \in[a, b] \subset(0,2 \alpha)$ and $s_{n}, \eta \in[c, d] \subset(0,2 \beta)$, for every $n$ $\in \mathbb{N}$ and suppose the following conditions hold:

(i) $\lim _{n \rightarrow \infty} \delta_{n}=\delta \in(0,1)$,

(ii) $0 \leq \kappa \leq \alpha_{n}<1, \forall n \geq 1$,

(iii) $\sum_{n=1}^{\infty}\left|\alpha_{1}^{n+1, j}-\alpha_{1}^{n, j}\right|<\infty, \sum_{n=1}^{\infty}\left|\alpha_{3}^{n+1, j}-\alpha_{3}^{n, j}\right|<\infty$, for all $j \in\{1,2,3, \ldots, N\}$.

(iv) There exists $\lambda_{1}, \lambda_{2}$ such that

$$
\left\{\begin{array}{l}
\Phi_{1}\left(w_{1}^{1}, T_{r_{1}}\left(x_{1}\right), T_{r_{2}}\left(x_{2}\right)\right)+\Phi_{1}\left(w_{2}^{1}, T_{r_{2}}\left(x_{2}\right), T_{r_{1}}\left(x_{1}\right)\right) \leq-\lambda_{1}\left\|T_{r_{1}}\left(x_{1}\right)-T_{r_{2}}\left(x_{2}\right)\right\|^{2} \text { and } \\
\Phi_{2}\left(w_{1}^{2}, T_{s_{1}}\left(x_{1}\right), T_{s_{2}}\left(x_{2}\right)\right)+\Phi_{2}\left(w_{2}^{2}, T_{s_{2}}\left(x_{2}\right), T_{s_{1}}\left(x_{1}\right)\right) \leq-\lambda_{2}\left\|T_{s_{1}}\left(x_{1}\right)-T_{s_{2}}\left(x_{2}\right)\right\|^{2}
\end{array}\right.
$$

for all $\left(r_{1}, r_{2}\right) \in \Theta \times \Theta,\left(s_{1}, s_{2}\right) \in \Xi \times \Xi, w_{i}^{1} \in T\left(x_{i}\right)$ and $w_{i}^{2} \in D\left(x_{i}\right)$, for $i=1,2$ where $\Theta=\left\{r_{n}: n \geq 1\right\}$ and $\Xi=\left\{s_{n}: n \geq 1\right\}$. Then $\left\{x_{n}\right\}$ converges strongly to $P_{\mathbb{E}} x_{1}$ which is a solution of (3.2):

$$
\left\{\begin{array}{l}
\left\langle A x^{*}, x-x^{*}\right\rangle \geq 0 \\
\left\langle B x^{*}, x-x^{*}\right\rangle \geq 0
\end{array}\right.
$$

Proof. From (3.1) for every $r \in \Theta$, we have

$$
\Phi_{1}\left(w_{1}^{1}, T_{r}\left(x_{1}\right), T_{r}\left(x_{2}\right)\right)+\Phi_{1}\left(w_{2}^{1}, T_{r}\left(x_{2}\right), T_{r}\left(x_{1}\right)\right) \leq-\lambda_{1}\left\|T_{r}\left(x_{1}\right)-T_{r}\left(x_{2}\right)\right\|^{2} \leq 0,
$$

for all $\left(x_{1}, x_{2}\right) \in C \times C$ and $w_{i}^{1} \in T\left(x_{i}\right), \quad i=1,2$.

Similarly, for every $s \in \Xi$, we have

$$
\Phi_{2}\left(w_{1}^{2}, T_{s}\left(x_{1}\right), T_{s}\left(x_{2}\right)\right)+\Phi_{2}\left(w_{2}^{2}, T_{s}\left(x_{2}\right), T_{s}\left(x_{1}\right)\right) \leq-\lambda_{2}\left\|T_{s}\left(x_{1}\right)-T_{s}\left(x_{2}\right)\right\|^{2} \leq 0 .
$$

for all $\left(x_{1}, x_{2}\right) \in C \times C$ and $w_{i}^{2} \in D\left(x_{i}\right), \quad i=1$, 2. From (3.3) and (3.4), we have Theorem 2.10 hold.

It is easy to see that $I-\lambda A$ and $I-\eta B$ are nonexpansive mapping. Indeed, since $A$ is a $\alpha$-inverse strongly monotone mapping with $\lambda \in(0,2 \alpha)$, we have 


$$
\begin{aligned}
\|(I-\lambda A) x-(I-\lambda A) y\|^{2} & =\|x-y-\lambda(A x-A y)\|^{2} \\
& =\|x-y\|^{2}-2 \lambda\langle x-y, A x-A y\rangle+\lambda^{2}\|A x-A y\|^{2} \\
& \leq\|x-y\|^{2}-2 \alpha \lambda\|A x-A y\|^{2}+\lambda^{2}\|A x-A y\|^{2} \\
& =\|x-y\|^{2}+\lambda(\lambda-2 \alpha)\|A x-A y\|^{2} \\
& \leq\|x-y\|^{2} .
\end{aligned}
$$

Thus $(I-\lambda A)$ is nonexpansive, so is $I-\eta B$. Since

$$
\Phi_{1}\left(w_{n}^{1}, u_{n}, u\right)+\varphi_{1}(u)-\varphi_{1}\left(u_{n}\right)+\frac{1}{r_{n}}\left\langle u_{n}-x_{n}, u-u_{n}\right\rangle \geq 0, \quad \forall u \in C,
$$

and Theorem 2.10, we have $u_{n}=T_{r_{n}} x_{n}$. Since

$$
\Phi_{2}\left(w_{n}^{2}, v_{n}, v\right)+\varphi_{2}(v)-\varphi_{2}\left(v_{n}\right)+\frac{1}{s_{n}}\left\langle v_{n}-x_{n}, v-v_{n}\right\rangle \geq 0, \quad \forall v \in C,
$$

and Theorem 2.10, we have $v_{n}=T_{s_{n}} x_{n}$. Let $z \in \mathbb{F}$, again by Theorem 2.10, we have $z=T_{r_{n}} z=T_{s_{n}} z=P_{C}(I-\lambda A) z=P_{C}(I-\eta B) z$. From nonexpansiveness of $\left\{T_{r_{n}}\right\},\left\{T_{S_{n}}\right\},\{I-\lambda A\}$, and $\{I-\eta B\}$, we have

$$
\begin{aligned}
\left\|z_{n}-z\right\| & =\left\|\delta_{n}\left(P_{C}(I-\lambda A) u_{n}-z\right)+\left(1-\delta_{n}\right)\left(P_{C}(I-\eta B) v_{n}-z\right)\right\| \\
& \leq \delta_{n}\left\|P_{C}(I-\lambda A) u_{n}-z\right\|+\left(1-\delta_{n}\right)\left\|P_{C}(I-\eta B) v_{n}-z\right\| \\
& \leq \delta_{n}\left\|T_{r_{n}} x_{n}-z\right\|+\left(1-\delta_{n}\right)\left\|T_{s_{n}} x_{n}-z\right\| \\
& \leq\left\|x_{n}-z\right\| .
\end{aligned}
$$

By (3.5), we have

$$
\begin{aligned}
\left\|y_{n}-z\right\| & =\left\|\alpha_{n}\left(z_{n}-z\right)+\left(1-\alpha_{n}\right)\left(S_{n} z_{n}-z\right)\right\| \\
& \leq \alpha_{n}\left\|z_{n}-z\right\|+\left(1-\alpha_{n}\right)\left\|S_{n} z_{n}-z\right\| \\
& \leq\left\|z_{n}-z\right\| \leq\left\|x_{n}-z\right\| .
\end{aligned}
$$

Next, we show that $C_{n}$ is closed and convex for every $n \in \mathbb{N}$. It is obvious that $C_{n}$ is closed. In fact, we know that, for $z \in C_{w}$

$$
\left\|y_{n}-z\right\| \leq\left\|x_{n}-z\right\| \text { is equivalent to }\left\|y_{n}-x_{n}\right\|^{2}+2\left\langle y_{n}-x_{n}, x_{n}-z\right\rangle \leq 0 .
$$

So, we have that $\forall z_{1}, z_{2} \in C_{n}$ and $t \in(0,1)$, it follows that

$$
\begin{aligned}
\left\|y_{n}-x_{n}\right\|^{2}+ & 2\left\langle y_{n}-x_{n}, x_{n}-\left(t z_{1}+(1-t) z_{2}\right)\right\rangle \\
= & t\left(2\left\langle y_{n}-x_{n}, x_{n}-z_{1}\right\rangle+\left\|y_{n}-x_{n}\right\|^{2}\right) \\
& +(1-t)\left(2\left\langle y_{n}-x_{n}, x_{n}-z_{2}\right\rangle+\left\|y_{n}-x_{n}\right\|^{2}\right) \\
\leq & 0,
\end{aligned}
$$

then, we have $C_{n}$ is convex. By Theorem 2.10 and Lemma 2.3, we conclude that $\mathbb{F}$ is closed and convex. This implies that $P_{\mathbb{F}}$ is well defined. Next, we show that $\mathbb{E} \subset C_{n}$ for every $n \in \mathbb{N}$. Putting $q \in \mathbb{F}$, by (3.6), it is easy to see that $q \in C_{n}$, then we have $\mathbb{F} \subset C_{n}$ for all $n \in \mathbb{N}$. Since $x_{n}=P_{C_{n}} x_{1}$, for every $w \in C_{n}$, we have

$$
\left\|x_{n}-x_{1}\right\| \leq\left\|w-x_{1}\right\|, \quad \forall n \in \mathbb{N} \text {. }
$$


In particular, we have

$$
\left\|x_{n}-x_{1}\right\| \leq\left\|P_{\mathbb{F}} x_{1}-x_{1}\right\| .
$$

Since $C$ is bounded, we have $\left\{x_{n}\right\}$ is bounded, so are $\left\{u_{n}\right\},\left\{v_{n}\right\},\left\{z_{n}\right\}$, and $\left\{y_{n}\right\}$. Since $x_{n+1}=P_{C_{n+1}} x_{1} \in C_{n+1} \subset C_{n}$ and $x_{n}=P_{C_{n}} x_{1}$, we have

$$
\begin{aligned}
0 & \leq\left\langle x_{1}-x_{n}, x_{n}-x_{n+1}\right\rangle \\
& =\left\langle x_{1}-x_{n}, x_{n}-x_{1}+x_{1}-x_{n+1}\right\rangle \\
& \leq-\left\|x_{n}-x_{1}\right\|^{2}+\left\|x_{n}-x_{1}\right\|\left\|x_{1}-x_{n+1}\right\|,
\end{aligned}
$$

it implies that

$$
\left\|x_{n}-x_{1}\right\| \leq\left\|x_{n+1}-x_{1}\right\| .
$$

Hence, we have $\lim _{n \rightarrow \infty}\left\|x_{n}-x_{1}\right\|$ exists. Since

$$
\begin{aligned}
\left\|x_{n}-x_{n+1}\right\|^{2} & =\left\|x_{n}-x_{1}+x_{1}-x_{n+1}\right\|^{2} \\
& =\left\|x_{n}-x_{1}\right\|^{2}+2\left\langle x_{n}-x_{1}, x_{1}-x_{n+1}\right\rangle+\left\|x_{1}-x_{n+1}\right\|^{2} \\
& =\left\|x_{n}-x_{1}\right\|^{2}+2\left\langle x_{n}-x_{1}, x_{1}-x_{n}+x_{n}-x_{n+1}\right\rangle+\left\|x_{1}-x_{n+1}\right\|^{2} \\
& =\left\|x_{n}-x_{1}\right\|^{2}-2\left\|x_{n}-x_{1}\right\|^{2}+2\left\langle x_{n}-x_{1}, x_{n}-x_{n+1}\right\rangle+\left\|x_{1}-x_{n+1}\right\|^{2} \\
& \leq\left\|x_{1}-x_{n+1}\right\|^{2}-\left\|x_{n}-x_{1}\right\|^{2},
\end{aligned}
$$

it implies that

$$
\lim _{n \rightarrow \infty}\left\|x_{n}-x_{n+1}\right\|=0 .
$$

Since $x_{n+1}=P_{C_{n+1}} x_{1} \in C_{n+1}$, we have

$$
\left\|y_{n}-x_{n+1}\right\| \leq\left\|x_{n}-x_{n+1}\right\|,
$$

by (3.10), we have

$$
\lim _{n \rightarrow \infty}\left\|y_{n}-x_{n+1}\right\|=0
$$

Since

$$
\left\|y_{n}-x_{n}\right\| \leq\left\|y_{n}-x_{n+1}\right\|+\left\|x_{n+1}-x_{n}\right\|,
$$

by (3.10) and (3.11), we have

$$
\lim _{n \rightarrow \infty}\left\|y_{n}-x_{n}\right\|=0 \text {. }
$$

Next, we show that

$$
\lim _{n \rightarrow \infty}\left\|z_{n}-S_{n} z_{n}\right\|=0 .
$$

By definition of $y_{n}$, we have

$$
y_{n}-z_{n}=\left(1-\alpha_{n}\right)\left(S_{n} z_{n}-z_{n}\right) .
$$

Claim that

$$
\lim _{n \rightarrow \infty}\left\|z_{n}-x_{n}\right\|=0 .
$$


Putting $M_{n}=P_{C}(I-\lambda A) u_{n}$ and $N_{n}=P_{C}(I-\eta B) v_{n}$, we have

$$
\left\|z_{n}-x_{n}\right\| \leq \delta_{n}\left\|M_{n}-x_{n}\right\|+\left(1-\delta_{n}\right)\left\|N_{n}-x_{n}\right\|
$$

Let $z \in \mathbb{F}$. Since $T_{r_{n}}$ is firmly nonexpansive mapping and $T_{r_{n}} x_{n}=u_{n}$, we have

$$
\begin{aligned}
\left\|z-u_{n}\right\|^{2} & =\left\|T_{r_{n}} z-T_{r_{n}} x_{n}\right\|^{2} \\
& \leq\left\langle T_{r_{n}} z-T_{r_{n}} x_{n}, z-x_{n}\right\rangle \\
& =\frac{1}{2}\left(\left\|u_{n}-z\right\|^{2}+\left\|x_{n}-z\right\|^{2}-\left\|u_{n}-x_{n}\right\|^{2}\right) .
\end{aligned}
$$

Hence

$$
\left\|u_{n}-z\right\|^{2} \leq\left\|x_{n}-z\right\|^{2}-\left\|u_{n}-x_{n}\right\|^{2}
$$

Since $T_{r_{n}}$ is firmly nonexpansive mapping and $T_{s_{n}} x_{n}=v_{n}$, by using the same method as (3.17), we have

$$
\left\|v_{n}-z\right\|^{2} \leq\left\|x_{n}-z\right\|^{2}-\left\|v_{n}-x_{n}\right\|^{2} .
$$

By nonexpansiveness of $S_{n}$ and (3.17), (3.18), we have

$$
\begin{aligned}
\left\|y_{n}-z\right\|^{2} & \leq\left\|z_{n}-z\right\|^{2} \\
& \leq \delta_{n}\left\|u_{n}-z\right\|^{2}+\left(1-\delta_{n}\right)\left\|v_{n}-z\right\|^{2} \\
& \leq \delta_{n}\left(\left\|x_{n}-z\right\|^{2}-\left\|u_{n}-x_{n}\right\|^{2}\right)+\left(1-\delta_{n}\right)\left(\left\|x_{n}-z\right\|^{2}-\left\|v_{n}-x_{n}\right\|^{2}\right) \\
& =\left\|x_{n}-z\right\|^{2}-\delta_{n}\left\|u_{n}-x_{n}\right\|^{2}-\left(1-\delta_{n}\right)\left\|v_{n}-x_{n}\right\|^{2},
\end{aligned}
$$

it implies that

$$
\begin{aligned}
\delta_{n}\left\|u_{n}-x_{n}\right\|^{2} & \leq\left\|x_{n}-z\right\|^{2}-\left\|y_{n}-z\right\|^{2}-\left(1-\delta_{n}\right)\left\|v_{n}-x_{n}\right\|^{2} \\
& \leq\left\|x_{n}-z\right\|^{2}-\left\|y_{n}-z\right\|^{2} \\
& \leq\left(\left\|x_{n}-z\right\|+\left\|y_{n}-z\right\|\right)\left\|x_{n}-y_{n}\right\|
\end{aligned}
$$

by (3.12) and condition (i), we have

$$
\lim _{n \rightarrow \infty}\left\|u_{n}-x_{n}\right\|=0 .
$$

By using the same method as (3.19), we have

$$
\lim _{n \rightarrow \infty}\left\|v_{n}-x_{n}\right\|=0 .
$$

Since

$$
\begin{aligned}
\left\|y_{n}-z\right\|^{2} \leq & \alpha_{n}\left\|z_{n}-z\right\|^{2}+\left(1-\alpha_{n}\right)\left\|z_{n}-z\right\|^{2} \\
\leq & \alpha_{n}\left\|x_{n}-z\right\|^{2}+\left(1-\alpha_{n}\right)\left\|z_{n}-z\right\|^{2} \\
\leq & \alpha_{n}\left\|x_{n}-z\right\|^{2}+\left(1-\alpha_{n}\right)\left(\delta_{n}\left\|M_{n}-z\right\|^{2}\right. \\
& \left.+(1-\delta)\left\|N_{n}-z\right\|^{2}\right)
\end{aligned}
$$

Claim that

$$
\lim _{n \rightarrow \infty}\left\|A u_{n}-A z\right\|=\lim _{n \rightarrow \infty}\left\|B v_{n}-B z\right\|=0 .
$$


By nonexpansiveness of $P_{C}$, we have

$$
\begin{aligned}
\left\|y_{n}-z\right\|^{2} \leq & \left\|z_{n}-z\right\|^{2} \\
\leq & \delta_{n}\left\|P_{C}(I-\lambda A) u_{n}-P_{C}(I-\lambda A) z\right\|^{2} \\
& +\left(1-\delta_{n}\right)\left\|P_{C}(I-\eta B) v_{n}-P_{C}(I-\eta B) z\right\|^{2} \\
\leq & \delta_{n}\left\|(I-\lambda A) u_{n}-(I-\lambda A) z\right\|^{2}+\left(1-\delta_{n}\right)\left\|(I-\eta B) v_{n}-(I-\eta B) z\right\|^{2} \\
\leq & \delta_{n} \|\left(u_{n}-\lambda A u_{n}-(z-\lambda A z)\left\|^{2}+\left(1-\delta_{n}\right)\right\|\left(v_{n}-\eta B v_{n}-(z-\eta B z) \|^{2}\right.\right. \\
= & \delta_{n}\left\|\left(u_{n}-z\right)-\lambda\left(A u_{n}-A z\right)\right\|^{2}+\left(1-\delta_{n}\right)\left\|\left(v_{n}-z\right)-\eta\left(B v_{n}-B z\right)\right\|^{2} \\
= & \delta_{n}\left(\left\|u_{n}-z\right\|^{2}+\lambda^{2}\left\|A u_{n}-A z\right\|^{2}-2 \lambda\left\langle u_{n}-z, A u_{n}-A z\right\rangle\right) \\
& +\left(1-\delta_{n}\right)\left(\left\|v_{n}-z\right\|^{2}+\eta^{2}\left\|B v_{n}-B z\right\|^{2}-2 \eta\left\langle v_{n}-z, B v_{n}-B z\right\rangle\right) \\
\leq & \delta_{n}\left(\left\|u_{n}-z\right\|^{2}+\lambda^{2}\left\|A u_{n}-A z\right\|^{2}-2 \lambda \alpha\left\|A u_{n}-A z\right\|^{2}\right) \\
& +\left(1-\delta_{n}\right)\left(\left\|v_{n}-z\right\|^{2}+\eta^{2}\left\|B v_{n}-B z\right\|^{2}-2 \eta \beta\left\|B v_{n}-B z\right\|^{2}\right) \\
\leq & \delta_{n}\left(\left\|x_{n}-z\right\|^{2}+\lambda(\lambda-2 \alpha)\left\|A u_{n}-A z\right\|^{2}\right) \\
& +\left(1-\delta_{n}\right)\left(\left\|x_{n}-z\right\|^{2}+\eta(\eta-2 \beta)\left\|B v_{n}-B z\right\|^{2}\right) \\
= & \left\|x_{n}-z\right\|^{2}-\delta_{n} \lambda(2 \alpha-\lambda)\left\|A u_{n}-A z\right\|^{2} \\
& -\left(1-\delta_{n}\right) \eta(2 \beta-\eta)\left\|B v_{n}-B z\right\|^{2},
\end{aligned}
$$

it follows that

$$
\begin{aligned}
\delta_{n} \lambda(2 \alpha-\lambda)\left\|A u_{n}-A z\right\|^{2} \leq & \left\|x_{n}-z\right\|^{2}-\left\|y_{n}-z\right\|^{2} \\
& -\left(1-\delta_{n}\right) \eta(2 \beta-\eta)\left\|B v_{n}-B z\right\|^{2} \\
\leq & \left(\left\|x_{n}-z\right\|+\left\|y_{n}-z\right\|\right)\left\|y_{n}-x_{n}\right\|
\end{aligned}
$$

by conditions (i), (ii), $\lambda \in(0,2 \alpha)$ and (3.12), it implies that

$$
\lim _{n \rightarrow \infty}\left\|A u_{n}-A z\right\|=0 .
$$

By using the same method as (3.23), we have

$$
\lim _{n \rightarrow \infty}\left\|B v_{n}-B z\right\|=0 .
$$

By nonexpansiveness of $T_{r_{n}}$, we have

$$
\begin{aligned}
\left\|M_{n}-z\right\|^{2}= & \left\|P_{C}\left(u_{n}-\lambda A u_{n}\right)-P_{C}(z-\lambda A z)\right\|^{2} \\
\leq & \left\langle\left(u_{n}-\lambda A u_{n}\right)-(z-\lambda A z), M_{n}-z\right\rangle \\
= & \frac{1}{2}\left(\left\|\left(u_{n}-\lambda A u_{n}\right)-(z-\lambda A z)\right\|^{2}+\left\|M_{n}-z\right\|^{2}-\|\left(u_{n}-\lambda A u_{n}\right)\right. \\
& \left.-(z-\lambda A z)-\left(M_{n}-z\right) \|^{2}\right) \\
\leq & \frac{1}{2}\left(\left\|u_{n}-z\right\|^{2}+\left\|M_{n}-z\right\|^{2}-\left\|\left(u_{n}-M_{n}\right)-\lambda\left(A u_{n}-A z\right)\right\|^{2}\right) \\
= & \frac{1}{2}\left(\left\|T_{r_{n}} x_{n}-T_{r_{n}} z\right\|^{2}+\left\|M_{n}-z\right\|^{2}-\left\|u_{n}-M_{n}\right\|^{2}\right. \\
& \left.+2 \lambda\left\langle u_{n}-M_{n}, A u_{n}-A z\right\rangle-\lambda^{2}\left\|A u_{n}-A z\right\|^{2}\right) \\
\leq & \frac{1}{2}\left(\left\|x_{n}-z\right\|^{2}+\left\|M_{n}-z\right\|^{2}-\left\|u_{n}-M_{n}\right\|^{2}+2 \lambda\left\langle u_{n}-M_{n}, A u_{n}-A z\right\rangle\right. \\
& \left.-\lambda^{2}\left\|A u_{n}-A z\right\|^{2}\right) .
\end{aligned}
$$


Hence, we have

$$
\begin{aligned}
\left\|M_{n}-z\right\|^{2} \leq & \left\|x_{n}-z\right\|^{2}-\left\|u_{n}-M_{n}\right\|^{2}+2 \lambda\left\langle u_{n}-M_{n}, A u_{n}-A z\right\rangle \\
& -\lambda^{2}\left\|A u_{n}-A z\right\|^{2} .
\end{aligned}
$$

By using the same method as (3.25), we have

$$
\left\|N_{n}-z\right\|^{2} \leq\left\|x_{n}-z\right\|^{2}-\left\|v_{n}-N_{n}\right\|^{2}+2 \eta\left\langle v_{n}-N_{n}, B v_{n}-B z\right\rangle-\eta^{2}\left\|B v_{n}-B z\right\|^{2} .
$$

Substitute (3.25) and (3.26) in (3.21), we have

$$
\begin{aligned}
\left\|y_{n}-z\right\|^{2} \leq & \alpha_{n}\left\|x_{n}-z\right\|^{2}+\left(1-\alpha_{n}\right)\left(\delta_{n}\left\|M_{n}-z\right\|^{2}\right. \\
& \left.+\left(1-\delta_{n}\right)\left\|N_{n}-z\right\|^{2}\right) \\
\leq & \alpha_{n}\left\|x_{n}-z\right\|^{2}+\left(1-\alpha_{n}\right)\left(\delta _ { n } \left(\left\|x_{n}-z\right\|^{2}-\left\|u_{n}-M_{n}\right\|^{2}\right.\right. \\
& \left.+2 \lambda\left\langle u_{n}-M_{n}, A u_{n}-A z\right\rangle-\lambda^{2}\left\|A u_{n}-A z\right\|^{2}\right)+\left(1+\delta_{n}\right)\left(\left\|x_{n}-z\right\|^{2}-\left\|v_{n}-N_{n}\right\|^{2}\right. \\
& \left.\left.+2 \eta\left\langle v_{n}-N_{n}, B v_{n}-B z\right\rangle-\eta^{2}\left\|B v_{n}-B z\right\|^{2}\right)\right) \\
\leq & \alpha_{n}\left\|x_{n}-z\right\|^{2}+\left(1-\alpha_{n}\right)\left(\delta_{n}\left\|x_{n}-z\right\|^{2}-\delta_{n}\left\|u_{n}-M_{n}\right\|^{2}\right. \\
& +2 \lambda \delta_{n}\left(u_{n}-M_{n}, A u_{n}-A z\right\rangle+\left(1-\delta_{n}\right)\left\|x_{n}-z\right\|^{2}-\left(1-\delta_{n}\right)\left\|v_{n}-N_{n}\right\|^{2} \\
& \left.+2 \eta\left(1-\delta_{n}\right)\left\langle v_{n}-N_{n}, B v_{n}-B z\right\rangle\right) \\
= & \alpha_{n}\left\|x_{n}-z\right\|^{2}+\left(1-\alpha_{n}\right)\left(\left\|x_{n}-z\right\|^{2}-\delta_{n}\left\|u_{n}-M_{n}\right\|^{2}\right. \\
& +2 \lambda \delta_{n}\left(u_{n}-M_{n}, A u_{n}-A z\right\rangle-\left(1-\delta_{n}\right)\left\|v_{n}-N_{n}\right\|^{2} \\
& \left.+2 \eta\left(1-\delta_{n}\right)\left\langle v_{n}-N_{n}, B v_{n}-B z\right\rangle\right) \\
= & \alpha_{n}\left\|x_{n}-z\right\|^{2}+\left(1-\alpha_{n}\right)\left\|x_{n}-z\right\|^{2}-\left(1-\alpha_{n}\right) \delta_{n}\left\|u_{n}-M_{n}\right\|^{2} \\
& +2\left(1-\alpha_{n}\right) \lambda \delta_{n}\left\langle u_{n}-M_{n}, A u_{n}-A z\right\rangle-\left(1-\delta_{n}\right)\left(1-\alpha_{n}\right)\left\|v_{n}-N_{n}\right\|^{2} \\
& +2 \eta\left(1-\delta_{n}\right)\left(1-\alpha_{n}\right)\left(v_{n}-N_{n}, B v_{n}-B z\right\rangle \\
= & \left\|x_{n}-z\right\|^{2}-\left(1-\alpha_{n}\right) \delta_{n}\left\|u_{n}-M_{n}\right\|^{2} \\
& +2\left(1-\alpha_{n}\right) \lambda \delta_{n}\left\langle u_{n}-M_{n}, A u_{n}-A z\right\rangle-\left(1-\delta_{n}\right)\left(1-\alpha_{n}\right)\left\|v_{n}-N_{n}\right\|^{2} \\
& +2 \eta\left(1-\delta_{n}\right)\left(1-\alpha_{n}\right)\left(v_{n}-N_{n}, B v_{n}-B z\right\rangle
\end{aligned}
$$

it implies that

$$
\begin{aligned}
\left(1-\alpha_{n}\right) \delta_{n}\left\|u_{n}-M_{n}\right\|^{2} \leq & \left\|x_{n}-z\right\|^{2}-\left\|y_{n}-z\right\|^{2} \\
& +2\left(1-\alpha_{n}\right) \lambda \delta_{n}\left\langle u_{n}-M_{n}, A u_{n}-A z\right\rangle-\left(1-\delta_{n}\right)\left(1-\alpha_{n}\right)\left\|v_{n}-N_{n}\right\|^{2} \\
& +2 \eta\left(1-\delta_{n}\right)\left(1-\alpha_{n}\right)\left\langle v_{n}-N_{n}, B v_{n}-B z\right\rangle \\
\leq & \left(\left\|x_{n}-z\right\|+\left\|y_{n}-z\right\|\right)\left\|y_{n}-x_{n}\right\| \\
& +2\left(1-\alpha_{n}\right) \lambda \delta_{n}\left\langle u_{n}-M_{n}, A u_{n}-A z\right\rangle \\
& +2 \eta\left(1-\delta_{n}\right)\left(1-\alpha_{n}\right)\left\langle v_{n}-N_{n}, B v_{n}-B z\right\rangle,
\end{aligned}
$$

from (3.12), (3.23), (3.24) and conditions (i) and (ii), we have

$$
\lim _{n \rightarrow \infty}\left\|u_{n}-M_{n}\right\|=0 .
$$

By using the same method as (3.28), we have

$$
\lim _{n \rightarrow \infty}\left\|v_{n}-N_{n}\right\|=0
$$

By (3.19) and (3.28), we have

$$
\lim _{n \rightarrow \infty}\left\|M_{n}-x_{n}\right\|=0 .
$$


By (3.20) and (3.29), we have

$$
\lim _{n \rightarrow \infty}\left\|N_{n}-x_{n}\right\|=0
$$

From (3.16), (3.30) and (3.31), we have

$$
\lim _{n \rightarrow \infty}\left\|z_{n}-x_{n}\right\|=0
$$

From (3.12) and (3.32), we have

$$
\lim _{n \rightarrow \infty}\left\|y_{n}-z_{n}\right\|=0
$$

From (3.14), (3.33) and condition (i), we have (3.13).

Let $a \in(0,1)$, by (3.10) there exists $N_{0} \in \mathbb{N}$ such

$$
\left\|x_{n+1}-x_{n}\right\|<a^{n}, \forall n \geq N_{0} .
$$

Thus, for any number $n, p \in \mathbb{N}, p>0$, we have

$$
\left\|x_{n+p}-x_{n}\right\| \leq \sum_{k=n}^{n+p-1}\left\|x_{k+1}-x_{k}\right\| \leq \sum_{k=n}^{n+p-1} a^{k} \leq \frac{a^{n}}{1-a} .
$$

Since $a \in(0,1)$, we have $\lim _{n \rightarrow \infty} a^{n}=0$. By (3.35), we have $\left\{x_{n}\right\}$ is Cauchy sequence in Hilbert space. Let $\lim _{n \rightarrow \infty} x_{n}=x^{*}$. Since $T: C \rightarrow C B(H)$ be $\mathcal{H}$-Lipschitz continuous with constant $\mu_{1}$ and (1.10), we have

$$
\left\|w_{n}^{1}-w_{n+1}^{1}\right\| \leq\left(1+\frac{1}{n}\right) \mathcal{H}\left(T\left(x_{n}\right), T\left(x_{n+1}\right)\right) \leq\left(1+\frac{1}{n}\right) \mu_{1}\left\|x_{n+1}-x_{n}\right\| .
$$

By (3.34) and for any number $n, p \in \mathbb{N}, p>0$, we have

$$
\begin{aligned}
\left\|w_{n+p}^{1}-w_{n}^{1}\right\| & \leq \sum_{k=n}^{n+p-1}\left\|w_{k+1}^{1}-w_{k}^{1}\right\| \\
& \leq \sum_{k=n}^{n+p-1}\left(1+\frac{1}{k}\right) \mu_{1}\left\|x_{k+1}-x_{k}\right\| \\
& \leq \sum_{k=n}^{n+p-1} 2 \mu_{1} a^{k} \\
& \leq 2 \mu_{1} \frac{a^{n}}{1-a} .
\end{aligned}
$$

Since $a \in(0,1)$, we have $\lim _{n \rightarrow \infty} a^{n}=0$. By (3.37), we have $\left\{w_{n}^{1}\right\}$ is cauchy sequence in Hilbert space. Let $\lim _{n \rightarrow \infty} w_{n}^{1}=w_{1}^{*}$. Next, we will prove that $w_{1}^{*} \in T\left(x^{*}\right)$. Since $w_{n}^{1} \in T\left(x_{n}\right)$, we have 


$$
\begin{aligned}
d\left(w_{n}^{1}, T\left(x^{*}\right)\right) & \leq \max \left\{d\left(w_{n}^{1}, T\left(x^{*}\right)\right), \sup _{w_{1} \in T\left(x^{*}\right)} d\left(T\left(x_{n}\right), w_{1}\right)\right\} \\
& \leq \max \left\{\sup _{z \in T\left(x_{n}\right)} d\left(z, T\left(x^{*}\right)\right), \sup _{w_{1} \in T\left(x^{*}\right)} d\left(T\left(x_{n}\right), w_{1}\right)\right\} \\
& =\mathcal{H}\left(T\left(x_{n}\right), T\left(x^{*}\right)\right) .
\end{aligned}
$$

Since

$$
\begin{aligned}
d\left(w_{1}^{*}, T\left(x^{*}\right)\right) & \leq\left\|w_{1}^{*}-w_{n}^{1}\right\|+d\left(w_{n}^{1}, T\left(x^{*}\right)\right) \\
& \leq\left\|w_{1}^{*}-w_{n}^{1}\right\|+\mathcal{H}\left(T\left(x_{n}\right), T\left(x^{*}\right)\right) \\
& \leq\left\|w_{1}^{*}-w_{n}^{1}\right\|+\mu_{1}\left\|x_{n}-x^{*}\right\|
\end{aligned}
$$

by $\lim _{n \rightarrow \infty} x_{n}=x^{*}$ and $\lim _{n \rightarrow \infty} w_{n}^{1}=w_{1}^{*}$, we have $d\left(w_{1}^{*}, T\left(x^{*}\right)\right)=0$, this implies that $w_{1}^{*} \in T\left(x^{*}\right)$. By using the same method as above, we have $\lim _{n \rightarrow \infty} w_{n}^{2}=w_{2}^{*}$ and $w_{2}^{*} \in D\left(x^{*}\right)$.

Let $\omega\left(x_{n}\right)$ be the set of all weakly $\omega$-limit of $\left\{x_{n}\right\}$. We shall show that $\omega\left(x_{n}\right) \subset \mathbb{F}$. Since $\left\{x_{n}\right\}$ is bounded, then $\omega\left(x_{n}\right) \neq 0$. Let $q \in \omega\left(x_{n}\right)$, there exists a subsequence $\left\{x_{n_{i}}\right\}$ of $\left\{x_{n}\right\}$ converse weakly to $q$. Since $\left\{x_{n}\right\}$ is a Cauchy sequence in Hilbert space, we have $x_{n_{i}} \rightarrow q$ as $\{i \rightarrow \infty\}$, it implies that $x_{n} \rightarrow q$ as $n \rightarrow \infty$. Since $\lim _{n \rightarrow \infty} x_{n}=x^{*}$ and $\lim _{n \rightarrow \infty} x_{n}=q$, we have $x^{*}=q$, then we have $w_{1}^{*} \in T(q)$ and $w_{2}^{*} \in D(q)$. From (3.19) and $x_{n} \rightarrow q$ as $n \rightarrow \infty$, we have $u_{n} \rightarrow q$ as $n \rightarrow \infty$.

By $u_{n}=T_{r_{n}} x_{n}$, we have

$$
\Phi_{1}\left(w_{n}^{1}, u_{n}, u\right)+\varphi_{1}(u)-\varphi_{1}\left(u_{n}\right)+\frac{1}{r_{n}}\left\langle u_{n}-x_{n}, u-u_{n}\right\rangle \geq 0, \quad \forall u \in C,
$$

by (3.19), (H1) and lower semicontinuity of $\phi_{1}$, we have

$$
\Phi_{1}\left(w_{1}^{*}, q, u\right)+\varphi_{1}(u)-\varphi_{1}(q) \geq 0, \forall u \in C,
$$

then, we have

$$
q \in(G E P)_{s}\left(\Phi_{1}, \varphi_{1}\right)
$$

By using the same method as (3.39), we have

$$
q \in(G E P)_{s}\left(\Phi_{2}, \varphi_{2}\right)
$$

Since $\kappa<a \leq \alpha_{1}^{n, j}, \alpha_{3}^{n, j} \leq b<1$ for all $j=1,2, \ldots, N-1, \kappa<c \leq \alpha_{1}^{n, N} \leq 1, \kappa \leq \alpha_{3}^{n, N} \leq d<1$ and $\kappa \leq \alpha_{2}^{n, j} \leq e<1$ for all $j=1,2, \ldots, N$. Without loss of generality, we may assume $\alpha_{1}^{n_{i} j} \rightarrow \alpha_{1}^{j} \in(\kappa, 1)$ as $i \rightarrow \infty, \alpha_{3}^{n_{i, j}} \rightarrow \alpha_{3}^{j} \in(\kappa, 1)$ and $\alpha_{2}^{n_{i, j}} \rightarrow \alpha_{2}^{j} \in(\kappa, 1)$ as $i \rightarrow \infty, \forall j=$ $1,2, \ldots, N$.

Let $S$ be $S$-mapping generated by $T_{1}, T_{2} \ldots, T_{N}$. and $\beta_{1}, \beta_{2}, \ldots, \beta_{N}$, where $\beta_{j}=\left(\alpha_{1}^{j}, \alpha_{2}^{j}, \alpha_{3}^{j}\right)$. By Lemma 2.4, we have $S$ is nonexpansive and $F(S)=\cap_{i=1}^{N} F\left(T_{i}\right)$. 
By Lemma 2.6, we have

$$
\lim _{k \rightarrow \infty}\left\|S{ }_{n_{i}} z_{n_{i}}-S z_{n_{i}}\right\|=0
$$

By (3.13) and (3.41), we have

$$
\lim _{n \rightarrow \infty}\left\|z_{n_{i}}-S z_{n_{i}}\right\|=0
$$

Since $x_{n_{i}} \rightarrow q$ as $i \rightarrow \infty$ and (3.32), we have $z_{n_{i}} \rightarrow q$ as $i \rightarrow \infty$. By $z_{n_{i}} \rightarrow q$ as $i \rightarrow \infty$, (3.42) and Lemma 2.7, we have

$$
q \in \bigcap_{i=1}^{N} F\left(T_{i}\right)
$$

Next, we define $Q: C \rightarrow C$ by

$$
Q x=\delta P_{C}(I-\lambda A) x+(1-\delta) P_{C}(I-\eta B) x .
$$

By Lemma 2.2, we have

$$
F(Q)=F\left(P_{C}(I-\lambda A)\right) \cap\left(P_{C}(I-\eta B)\right)=F\left(G_{1}\right) \cap F\left(G_{2}\right) .
$$

From (3.44), we have

$$
\begin{aligned}
\left\|Q x_{n}-x_{n}\right\| \leq & \left\|Q x_{n}-z_{n}\right\|+\left\|z_{n}-x_{n}\right\| \\
\leq & \| \delta P_{C}(I-\lambda A) x_{n}+(1-\delta) P_{C}(I-\eta B) x_{n} \\
& -\delta_{n} P_{C}(I-\lambda A) u_{n}-\left(1-\delta_{n}\right) P_{C}(I-\eta B) v_{n}\|+\| z_{n}-x_{n} \| \\
= & \| \delta P_{C}(I-\lambda A) x_{n}-\delta P_{C}(I-\lambda A) u_{n}+\delta P_{C}(I-\lambda A) u_{n} \\
& +(1-\delta) P_{C}(I-\eta B) x_{n}-(1-\delta) P_{C}(I-\eta B) v_{n}+(1-\delta) P_{C}(I-\eta B) v_{n} \\
& -\delta_{n} P_{C}(I-\lambda A) u_{n}-\left(1-\delta_{n}\right) P_{C}(I-\eta B) v_{n}\|+\| z_{n}-x_{n} \| \\
\leq & \delta\left\|P_{C}(I-\lambda A) x_{n}-P_{C}(I-\lambda A) u_{n}\right\|+\left|\delta-\delta_{n}\right|\left\|P_{C}(I-\lambda A) u_{n}\right\| \\
& +(1-\delta)\left\|P_{C}(I-\eta B) x_{n}-P_{C}(I-\eta B) v_{n}\right\|+\left|(1-\delta)-\left(1-\delta_{n}\right)\right|\left\|P_{C}(I-\eta B) v_{n}\right\| \\
& +\left\|z_{n}-x_{n}\right\| \\
\leq & \delta \\
& \left\|x_{n}-u_{n}\right\|+\left|\delta-\delta_{n}\right|\left\|P_{C}(I-\lambda A) u_{n}\right\| \\
& +(1-\delta)\left\|x_{n}-v_{n}\right\|+\left|\delta_{n}-\delta\right|\left\|P_{C}(I-\eta B) v_{n}\right\| \\
& +\left\|z_{n}-x_{n}\right\|
\end{aligned}
$$

by condition (i), (3.19), (3.20), and (3.32), we have

$$
\lim _{n \rightarrow \infty}\left\|Q x_{n}-x_{n}\right\|=0
$$

Since $x_{n_{i}} \rightarrow q$ as $i \rightarrow \infty$ and Lemma 2.7, we have

$$
q \in F(Q)=F\left(G_{1}\right) \cap F\left(G_{2}\right) .
$$

From (3.39), (3.40), (3.43), and (3.47), we have $q \in \mathbb{F}$. Hence $\omega\left(x_{n}\right) \subset \mathbb{F}$. Hence, by Lemma 2.8 and (3.8), it implies that $\left\{x_{n}\right\}$ converges strongly to $P_{\mathbb{F}} x_{1}$. This completes the proof.

Remark 3.2. If we take $T \equiv D, w_{n}^{1}=w_{n}^{2} u_{n}=v_{n} \forall n \in \mathbb{N}, \Phi_{1} \equiv \Phi_{2}$ and $\phi_{1}=\phi_{2}$, then the Algorithm 1.3 reduces to the following algorithm: 


$$
\left\{\begin{array}{l}
w_{n}^{1} \in T\left(x_{n}\right),\left\|w_{n}^{1}-w_{n+1}^{1}\right\| \leq\left(1+\frac{1}{n}\right) \mathcal{H}\left(T\left(x_{n}\right), T\left(x_{n+1}\right)\right), \\
\Phi_{1}\left(w_{n}^{1}, u_{n}, u\right)+\varphi_{1}(u)-\varphi_{1}\left(u_{n}\right)+\frac{1}{r_{n}}\left\langle u_{n}-x_{n}, u-u_{n}\right\rangle \geq 0, \forall u \in C, \\
z_{n}=P_{C}(I-\lambda A) u_{n} \\
y_{n}=\alpha_{n} z_{n}+\left(1-\alpha_{n}\right) S_{n} z_{n} \\
C_{n+1}=\left\{z \in C_{n+1}:\left\|y_{n}-z\right\| \leq\left\|x_{n}-z\right\|\right\} \\
x_{n+1}=P_{C_{n+1}} x_{1}, \forall n \geq 1
\end{array}\right.
$$

under the same conditions of Theorem 3.1, we have the sequence $\left\{x_{n}\right\}$ generated by algorithm (3.48) converges strongly to $P_{\mathbb{F}} x_{1}$ where $\mathbb{F}=\cap_{i=1}^{N} F\left(T_{i}\right) \cap(G E P)_{s}\left(\Phi_{1}, \varphi_{1}\right) \cap F\left(G_{1}\right)$, where $G_{1}: C \rightarrow C$ is defined by $G_{1}(x)=P_{C}$ $(x-\lambda A x) \forall x \in C$ and $P_{\mathbb{F}} x_{1}$ is a solution of $\left\langle A x^{*}, x-x^{*}\right\rangle \geq 0$

\section{Application}

In this section, by using our main result we prove a strong convergence theorem of the sequence $\left\{x_{n}\right\}$ generated by Algorithm 4.1 as follows:

Algorithm 4.1. Let $T_{i}, i=1,2, \ldots, N$, be $\kappa_{i}$-pseudo contraction mappings of $C$ into itself and $\kappa=\max \left\{\kappa_{i}: i=1,2, \ldots, N\right\}$ and let $S_{n}$ be the $S$-mappings generated by $T_{1}, T_{2}, \ldots, T_{N}$ and $\alpha_{1}^{(n)}, \alpha_{2}^{(n)}, \ldots, \alpha_{N}^{(n)}$ where $\alpha_{j}^{(n)}=\left(\alpha_{1}^{n, j}, \alpha_{2}^{n, j}, \alpha_{3}^{n, j}\right) \in I \times I \times I, I=[0,1], \alpha_{1}^{n, j}+\alpha_{2}^{n, j}+\alpha_{3}^{n, j}=1$ and $\kappa<a \leq \alpha_{1}^{n, j}, \alpha_{3}^{n, j} \leq b<1$ for all $j=1,2, \ldots, N-1, \kappa<c \leq \alpha_{1}^{n, N} \leq 1, \kappa \leq \alpha_{3}^{n, N} \leq d<1, \kappa \leq \alpha_{2}^{n, j} \leq e<1$ for all $j=1,2, \ldots, N$. Let $x_{1} \in C=C_{1}$ and $w_{1}^{i} \in T^{i}\left(x_{1}\right)$, there exists sequence $\left\{w_{n}^{i}\right\} \in H$ and $\left\{x_{n}\right\},\left\{u_{n}^{i}\right\} \subseteq C, \forall i=1,2, \ldots, N$ such that

$$
\left\{\begin{array}{l}
w_{n}^{i} \in T^{i}\left(x_{n}\right),\left\|w_{n}^{i}-w_{n+1}^{i}\right\| \leq\left(1+\frac{1}{n}\right) \mathcal{H}\left(T\left(x_{n}\right), T\left(x_{n+1}\right)\right) \forall i=1,2, \ldots, N \\
\Phi_{i}\left(w_{n}^{i}, u_{n}^{i}, u\right)+\varphi_{i}(u)-\varphi_{i}\left(u_{n}^{i}\right)+\frac{1}{r_{n}^{i}}\left\langle u_{n}^{i}-x_{n}, u-u_{n}^{i}\right\rangle \geq 0, \forall u \in C, \quad \forall i=1,2, \ldots, N \\
z_{n}=\sum_{i=1}^{N} \delta_{n}^{i} P_{C}\left(I-\lambda_{i} A_{i}\right) u_{n}^{i}, \text { where } \sum_{i=1}^{N} \delta_{n}^{i}=1 \\
y_{n}=\alpha_{n} z_{n}+\left(1-\alpha_{n}\right) S_{n} z_{n} \\
C_{n+1}=\left\{z \in C_{n+1}:\left\|y_{n}-z\right\| \leq\left\|x_{n}-z\right\|\right\} \\
x_{n+1}=P_{C_{n+1}} x_{1}, \forall n \geq 1
\end{array}\right.
$$

The following result can be obtained from Theorem 3.1. We, therefore, omit the proof.

Theorem 4.2. Let $C$ be a nonempty bounded, closed, and convex subset of Hilbert space $H$ and let $\phi_{i}$ : be a lower semicontinuous and convex function, for all $i=1,2, \ldots, N$. Let $T^{i}: C$ $\rightarrow C B(H)$ be $\mathcal{H}$-Lipschitz continuous with constant $\mu_{i}, \Phi_{i}: H \times C \times C \rightarrow \mathbb{R}$ be equilibrium-like functions satisfying $(H 1)-(H 3)$ and $A_{i}: C \rightarrow H$ be a $\alpha_{i}$-inverse strongly monotone mappings $\forall i=1,2, \ldots, N$ and let $T_{i}, i=1,2, \ldots, N$, be $\kappa_{i}$-pseudo contraction mappings of $C$ into itself and $\kappa=\max \left\{\kappa_{i}: i=1,2, \ldots, N\right\}$ with $\mathbb{F}=\cap_{i=1}^{N} F\left(T_{i}\right) \cap_{i=1}^{N}(G E P)_{s}\left(\Phi_{i}, \varphi_{i}\right) \cap_{i=1}^{N} F\left(G_{i}\right)$, where $G_{i}: C \rightarrow C$ is defined by $G_{i}(x)=P_{C}\left(x-\lambda_{i} A_{i} x\right) \forall x \in C, i=1,2, \ldots, N$. Let $S_{n}$ be the $S$ mappings generated by $T_{1}, \quad T_{2}, \ldots, \quad T_{N}$ and $\alpha_{1}^{(n)}, \alpha_{2}^{(n)}, \ldots, \alpha_{N}^{(n)}$ where $\alpha_{j}^{(n)}=\left(\alpha_{1}^{n, j}, \alpha_{2}^{n, j}, \alpha_{3}^{n, j}\right) \in I \times I \times I, I=[0,1], \alpha_{1}^{n, j}+\alpha_{2}^{n, j}+\alpha_{3}^{n, j}=1 \quad$ and $\kappa<a \leq \alpha_{1}^{n, j}, \alpha_{3}^{n, j} \leq b<1 \quad$ for all $j=1,2, \ldots, N-1, \kappa<c \leq \alpha_{1}^{n, N} \leq 1, \kappa \leq \alpha_{3}^{n, N} \leq d<1, \kappa \leq \alpha_{2}^{n, j} \leq e<1$ for all $j=1,2, \ldots$, $N$ and let $\left\{x_{n}\right\},\left\{u_{n}^{i}\right\},\left\{w_{n}^{i}\right\}, \quad \forall i=1,2, \ldots, N$, be sequences generated by (4.1), where $\left\{\alpha_{n}\right\}$ is 
a sequence in $[0,1], r_{n}^{i}, \lambda_{i} \in[a, b] \subset(0,2 \alpha) \forall i=1,2, \ldots, N$ and $n \in \mathbb{N}$ and suppose the following conditions hold:

(i) $\lim _{n \rightarrow \infty} \delta_{n}^{i}=\delta^{i} \in(0,1), \forall i=1,2, \ldots, N$.

(ii) $0 \leq \kappa \leq \alpha_{n}<1, \forall n \geq 1$,

(iii) $\sum_{n=1}^{\infty}\left|\alpha_{1}^{n+1, j}-\alpha_{1}^{n, j}\right|<\infty, \sum_{n=1}^{\infty}\left|\alpha_{3}^{n+1, j}-\alpha_{3}^{n, j}\right|<\infty$, for all $j \in\{1,2, \ldots, N\}$.

(iv) There exists $\lambda^{i}, \forall i=1,2, \ldots, N$ such that

$$
\Phi_{1}\left(w_{1}^{i}, T_{r_{1}^{i}}\left(x_{1}\right), T_{r_{2}^{i}}\left(x_{2}\right)\right)+\Phi_{1}\left(w_{2}^{i}, T_{r_{2}^{i}}\left(x_{2}\right), T_{r_{1}^{i}}\left(x_{1}\right)\right) \leq-\lambda^{i}\left\|T_{r_{1}^{i}}\left(x_{1}\right)-T_{r_{2}^{i}}\left(x_{2}\right)\right\|^{2},
$$

for all $i=1,2, \ldots, N,\left(r_{1}^{i}, r_{2}^{i}\right) \in \Theta^{i} \times \Theta^{i}, w_{k}^{i} \in T^{i}\left(x_{k}\right) \quad$ for $k=1,2$ where $\Theta^{i}=\left\{r_{n}^{i}: n \geq 1\right\}$. Then $\left\{x_{n}\right\}$ converges strongly to $P_{\mathbb{F}} x_{1}$ and $P_{\mathbb{F}} x_{1}$ is a solutions of (4.3):

$$
\left\langle A_{i} x^{*}, x-x^{*}\right\rangle \geq 0, \forall i=1,2, \ldots, N \text {. }
$$

\section{Competing interests}

The author declares that they have no competing interests.

Received: 26 September 2011 Accepted: 28 February 2012 Published: 28 February 2012

\section{References}

1. Goebel, K, Kirk, WA: Topics in Metric Fixed Point Theory. In Cambridge Stud Adv Math, vol. 28,Cambridge University Press, Cambridge (1990)

2. liduka, H, Takahashi, W: Weak convergence theorem by Ces'aro means for nonexpansive mappings and inverse-strongly monotone mappings. J Nonlinear Convex Anal. 7, 105-113 (2006)

3. Combettes, PL, Hirstoaga, SA: Equilibrium programming in Hilbert spaces. J Nonlinear Convex Anal. 6(1):117-136 (2005)

4. Blum, E, Oettli, W: From optimization and variational inequalities to equilibrium problems. Math Student. 63(14):123-145 (1994)

5. Blum, E, Oettli, W: From optimization and variational inequalities to equilibrium problems. Math Stud. 63, 123-145 (1994)

6. Moudafi, A: Weak convergence theorems for nonexpansive mappings and equilibrium problems. J Nonlinear Convex Anal. 9, 37-43 (2008)

7. He, H, Liu, S, Cho, YJ: An explicit method for systems of equilibrium problems and fixed points of infinite family of nonexpansive mappings. J Comput Appl Math. 235, 4128-4139 (2011). doi:10.1016/j.cam.2011.03.003

8. Yao, Y, Cho, YJ, Liou, YC: Iterative algorithms for variational inclusions, mixed equilibrium problems and fixed point problems approach to optimization problems. Cent Eur J Math. 9, 640-656 (2011). doi:10.2478/s11533-011-0021-3

9. Yao, Y, Cho, YJ, Liou, YC: Algorithms of common solutions for varia-tional inclusions, mixed equilibrium problems and fixed point problems. Eur J Oper Res. 212, 242-250 (2011). doi:10.1016/j.ejor.2011.01.042

10. Cho, YJ, Argyros, IK, Petrot, N: Approximation methods for common solutions of generalized equilibrium, systems of nonlinear variational inequalities and fixed point problems. Comput Math Appl. 60, 2292-2301 (2010). doi:10.1016/j. camwa.2010.08.021

11. Cho, YJ, Petrot, N: An Optimization problem related to generalized equilibrium and fixed point problems with applications. Fixed Point Theory. 11, 237-250 (2010)

12. Qin, $X$, Chang, Ss, Cho, YJ: Iterative methods for generalized equilibrium problems and fixed point problems with applications. Nonlinear Anal: Real World Appl. 11, 2963-2972 (2010). doi:10.1016/j.nonrwa.2009.10.017

13. Cho, YJ, Petrot, N: On the system of nonlinear mixed implicit equilibrium problems in Hilbert spaces. J Inequal Appl 2010, 12 (2010). Article ID 437976

14. Qin, X, Cho, YJ, Kang, SM: Viscosity approximation methods for generalized equilibrium problems and fixed point problems with applications. Nonlinear Anal. 72, 99-112 (2010). doi:10.1016/j.na.2009.06.042

15. Qin, X, Cho, YJ, Kang, SM: Convergence analysis on hybrid projection algorithms for equilibrium problems and variational inequality problems. Math Model Anal. 14, 335-351 (2009). doi:10.3846/1392-6292.2009.14.335-351

16. Cho, YJ, Kang, JI, Qin, X: Convergence theorems based on hybrid methods for generalized equilibrium problems and fixed point problems. Nonlinear Anal. 71, 4203-4214 (2009). doi:10.1016/i.na.2009.02.106

17. Ceng, LC, Ansari, QH, Yao, JC: Viscosity approximation methods for generalized equilibrium problems and fixed point problems. J Glob Optim. 43, 487-502 (2009). doi:10.1007/s10898-008-9342-6

18. Kangtunyakarn, A: Hybrid algorithm for finding common elements of the set of generalized equilibrium problems and the set of fixed point problems of strictly pseudocontractive mapping. Fixed Point Theory and Applications 2011, 19 (2011). Article ID 274820. doi:10.1186/1687-1812-2011-19

19. Takahashi, W: Nonlinear Functional Analysis. Yokohama Publishers, Yokohama (2000)

20. Bruck, RE: Properties of fixed point sets of nonexpansive mappings in Banach spaces. Trans Am Math Soc. 179, 251-262 (1973) 
21. Kangtunyakarn, A, Suantai, S: Strong convergence of a new iterative scheme for a finite family of strict pseudocontractions. Comput Math Appl. 60, 680-694 (2010). doi:10.1016/j.camwa.2010.05.016

22. Marino, G, Xu, HK: Weak and strong convergence theorems for strict pseudo-contractions in Hilbert spaces. J Math Anal Appl. 329, 336-346 (2007). doi:10.1016/j.jmaa.2006.06.055

23. Browder, FE: Nonlinear operators and nonlinear equations of evolution in Banach spaces. Proc Sympos Pure Math. 18 78-81 (1976)

24. Matines-Yanes, $\mathrm{C}, \mathrm{Xu}, \mathrm{HK}$ : Strong convergence of the CQ method for fixed point iteration processes. Nonlinear Anal. 64 , 2400-2411 (2006). doi:10.1016/j.na.2005.08.018

25. Nadler, SB Jr: Multivalued contraction mappings. Pacific J Math. 30, 475-488 (1969)

26. Takahashi, W: Nolinear and Convex Analysis. Yokohama Publishers, Yokohama (2009)

doi:10.1186/1687-1812-2012-30

Cite this article as: Kangtunyakarn: Hybrid iterative scheme for a generalized equilibrium problems, variational inequality problems and fixed point problem of a finite family of $\kappa_{i}$-strictly pseudocontractive mappings. Fixed Point Theory and Applications 2012 2012:30.

\section{Submit your manuscript to a SpringerOpen ${ }^{\circ}$} journal and benefit from:

- Convenient online submission

- Rigorous peer review

- Immediate publication on acceptance

- Open access: articles freely available online

- High visibility within the field

- Retaining the copyright to your article

Submit your next manuscript at $\gg$ springeropen.com 